\title{
Input-to-State Stability for cascade systems with multiple invariant sets
}

\author{
Paolo Forni ${ }^{\mathrm{a}}$, David Angeli ${ }^{\mathrm{b}}$ \\ ${ }^{a}$ Dept. of Electrical and Electronic Engineering, Imperial College London, UK \\ ${ }^{b}$ Dept. of Electrical and Electronic Engineering, Imperial College London, UK, and Dip. di Ingegneria dellInformazione of University of
} Florence, Italy

\begin{abstract}
In a recent paper [2], the notion of Input-to-State Stability (ISS) has been generalized for systems with decomposable invariant sets and evolving on Riemannian manifolds. In this work, we analyze the cascade interconnection of such ISS systems and we characterize the finest possible decomposition of its invariant set for three different scenarios: 1 . the driving system exhibits multistability (convergence to fixed points only); 2. the driving system exhibits multi-almost periodicity (convergence to fixed points as well as periodic and almost-periodic orbits) and the driven system is assumed to be incremental ISS; 3. the driving system exhibits multiperiodicity (convergence to fixed points and periodic orbits) whereas the driven system is ISS in the sense of [2]. Furthermore, we provide marginal results on the backward/forward asymptotic behavior of incremental ISS systems and on the response of a contractive system under asymptotically almost-periodic forcing. Three examples illustrate the potentiality of the proposed framework.
\end{abstract}

\section{Keywords:}

Input-to-State Stability, Lyapunov methods, interconnections.

\section{Introduction}

Input-to-State Stability (ISS) has been proven a very meaningful notion of stability and sensitivity to disturbances for nonlinear systems [15]. Apart from being a tool for the analysis, ISS has had a central role in the design of nonlinear feedback systems, with applications ranging from feedback redesign, small-gain theorems, tracking design, observers, and stabilization under saturated feedback. One of the major advances in this direction is the stabilization of nonlinear cascades, whose recursive application led to several constructive design methods such as backstepping and forwarding [12]. Indeed, in many cases of interest, the cascaded decomposition of the system under consideration is advantageous in providing the explicit stabilizing feedback law. Moreover, the ISS property behaves well under composition: a cascade of ISS system is again ISS, under suitable dissipation rates and gain functions of the driving/driven system, see [13].

Recently, a generalization of ISS theory for systems with decomposable invariant sets and evolving on Riemannian manifolds [2] has allowed the stability analysis in presence of inputs for a broader variety of systems exhibiting many dynamical behaviors of interests, such as multistability, periodic oscillations, almost global asymptotic stability, just to name a few. In this new setting, the decomposable invariant sets are no longer required to satisfy the Lyapunov stability requirement as long as they

Email addresses: p.forni14@imperial.ac.uk (Paolo Forni), d.angeli@imperial.ac.uk (David Angeli) retain the global attractivity property and admit a decomposition without cycles, as specified in Definitions 2.1, 2.2, and 2.3 (basically no homoclinic nor heteroclinic orbits may exist).

Largely inspired by the applications in biological (see the mitogen-activated protein kinase (MAPK) as an example of cascade) as well as mechanical networks, in this work we study nonlinear cascades of systems belonging to the class described above, so that the novel generalized ISS theory can be applied. Not surprisingly, the ISS property is still conserved under cascade interconnection, under the implicit requirement to specify a compact invariant set for the cascade which is globally attractive and admits a decomposition without cycles. In particular, we characterize the finest possible decomposition of such invariant set in three different scenarios. In the first one, the driving system is assumed to exhibit multistable behavior, that is asymptotic convergence of all trajectories to fixed points only; the results provided by Thieme [16] for asymptotically autonomous semiflows turn out to be crucial in the analysis of this setting. In the second scenario, the driving system is assumed to have fixed points as well as periodic orbits and almost-periodic attractors (multialmost periodicity), whereas the driven system is assumed to satisfy the incremental ISS property [1]. Indeed, incremental ISS is a very natural option for the analysis of this scenario. In the third scenario, the incremental ISS requirement for the driving system is relaxed to only ISS in the sense of [2]. It is within latter scenario that inferring ISS of the cascades comprises particularly novel results 
concerning the so-called converging-input-converging-state (CICS) $[10,14]$ for systems under asymptotically periodic forcing.

The rest of the paper is organized as follows. Section 2 introduces the notion of decomposable invariant set and the class of cascade systems under consideration. Sections 3 and 4 respectively address the first and second scenarios (multistability and multi-almost periodicity). The third scenario is studied in Section 5. Section 6 collects examples for all three aforemention scenarios. Final remarks are collected in Section 7.

Notation. Symbol $\mathfrak{d}\left(w_{1}, w_{2}\right)$ denotes the Riemannian distance between $w_{1}, w_{2} \in M_{w}$. For a point $w \in M_{w}$, and for a subset $S \subset M_{w}$, the set-point distance is defined as:

$$
|w|_{S}=\inf _{a \in S} \mathfrak{d}(w, a) .
$$

We define the equivalent of the infinity norm of the distance of signals taking values on $M_{w}$ as follows:

$$
\mathfrak{d}_{[a, b]}\left(X_{1}(\cdot), X_{2}(\cdot)\right):=\sup _{t \in[a, b]} \mathfrak{d}\left(X_{1}(t), X_{2}(t)\right) .
$$

Notation $|\cdot|$ indicates the standard Euclidean norm. For a measurable function $d: \mathbb{R}_{+} \rightarrow \mathbb{R}^{m}$ we define its infinity norm over the time interval $\left[t_{1}, t_{2}\right]$ as $\left\|d_{\left[t_{1}, t_{2}\right]}\right\|=$

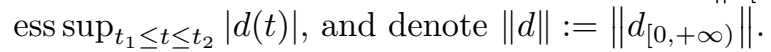

\section{Definitions and main assumptions}

\subsection{Decompositions and ISS for multistable systems}

In this Section we will introduce the notion of decomposition of a compact invariant set of a nonlinear dynamical system. In fact, as pointed out in [2], the decomposition of a compact invariant set of a nonlinear system exhibiting neither homoclinic nor heteroclinic cycles plays a crucial role when claiming ISS. Moreover, it can be observed that such assumption automatically rules out a number of conservative systems (for instance, Hamiltonian systems).

Let $M$ be an $n$-dimensional connected and geodesically complete Riemannian manifold without boundary. Let $D$ be a closed subset of $\mathbb{R}^{m}$ containing the origin. Consider the system:

$$
\dot{w}(t)=F(w(t), d(t)),
$$

where $F(w, d): M \times D \rightarrow T_{x} M$ is a locally Lipschitz continuous mapping with state $w$ taking value in $M$ and $d(\cdot)$ any locally essentially bounded and measurable input signal taking values in $D$. We denote by $W(t, w ; d)$ the uniquely defined solution of (1) at time $t$ fulfilling $W(0, w ; d)=w$ under the input $d(\cdot)$.

The unperturbed system is defined by the following set of equations:

$$
\dot{w}(t)=F(w(t), 0) .
$$

We assume that all solutions of (2) are complete ${ }^{1}$ and that all (possibly empty) $\alpha$ - and $\omega$-limit sets are compact.

\footnotetext{
${ }^{1}$ Without loss of generality, system (1) can be made backward and forward complete by slowing down the dynamics with $\dot{w}=$ $\frac{1}{1+|F(w, d)|_{\mathfrak{g}}} F(w, d)$, where $\mathfrak{g}$ denotes the Riemannian metric on $M$.
}

Definition 2.1 ( $\mathcal{W}$-limit set). Let $\mathcal{W}_{w} \subset M$ be a compact invariant set containing all the $\alpha$-and $\omega$-limit sets of (2), i.e. $\alpha(w) \cup \omega(w) \subseteq \mathcal{W}_{w}$ for all $w \in M$. Then the set $\mathcal{W}_{w}$ is called an $\mathcal{W}$-limit set for (2).

Definition 2.2 (Decomposition). Let $\mathcal{W}_{w} \in M$ be a compact and invariant set for (2). A decomposition of $\mathcal{W}_{w}$ is a finite, disjoint family of compact invariant sets $\mathcal{W}_{w, 1}$, $\ldots, \mathcal{W}_{w, K}$ (the atoms of the decomposition) such that:

$$
\mathcal{W}_{w}=\bigcup_{i=1}^{K} \mathcal{W}_{w, i}
$$

For an invariant set $\mathcal{W}_{w}$, its attracting and repulsing subsets are defined as follows:

$$
\begin{aligned}
& \mathcal{A}\left(\mathcal{W}_{w}\right)=\left\{w \in M_{w}:|W(t, w, 0)|_{\mathcal{W}_{w}} \rightarrow 0 \text { as } t \rightarrow+\infty\right\}, \\
& \mathcal{R}\left(\mathcal{W}_{w}\right)=\left\{w \in M_{w}:|W(t, w, 0)|_{\mathcal{W}_{w}} \rightarrow 0 \text { as } t \rightarrow-\infty\right\} .
\end{aligned}
$$

Define a relation on $\mathcal{W}_{w, i}$ and $\mathcal{W}_{w, j}$ by $\mathcal{W}_{w, i} \prec \mathcal{W}_{w, j}$ if $\mathcal{A}\left(\mathcal{W}_{w, i}\right) \cap \mathcal{R}\left(\mathcal{W}_{w, j}\right) \neq \emptyset$.

Definition 2.3 ( $r$-cycle, 1-cycle, filtration). Let $\mathcal{W}_{w, 1}, \ldots$, $\mathcal{W}_{w, K}$ be a decomposition of $\mathcal{W}_{w}$, then:

1. An $r$-cycle $(r \geq 2)$ is an ordered $r$-tuple of distinct indexes $i_{1}, \ldots, i_{r}$ such that $\mathcal{W}_{w, i_{1}} \prec \cdots \prec \mathcal{W}_{w, i_{r}} \prec$ $\mathcal{W}_{w, i_{1}}$.

2. A 1-cycle is an index $i$ such that $\left[\mathcal{R}\left(\mathcal{W}_{w, i}\right) \cap \mathcal{A}\left(\mathcal{W}_{w, i}\right)\right]$ $\backslash \mathcal{W}_{w, i} \neq \emptyset$.

3. A filtration ordering is a numbering of the $\mathcal{W}_{w, i}$ so that $\mathcal{W}_{w, i} \prec \mathcal{W}_{w, j} \Rightarrow i \leq j$.

Existence of an $r$-cycle for (2) with $r \geq 2$ is equivalent to existence of a heteroclinic cycle, and existence of a 1cycle implies existence of a homoclinic orbit.

Definition 2.4 (No cycle condition). The autonomous system (2) is said to satisfy the no-cycle condition if it has an $\mathcal{W}$-limit set $\mathcal{W}_{w}$ as in Definition 2.1 that admits a finite decomposition without cycles, namely $\mathcal{W}_{w}=\bigcup_{i=1}^{K} \mathcal{W}_{w, i}$ for some non-empty disjoint compact sets $\mathcal{W}_{w, i}$, which form a filtration ordering of $\mathcal{W}_{w}$, as detailed in Definitions 2.2 and 2.3. Under the specified assumptions, the set $\mathcal{W}_{w}$ is said to satisfy the no-cycle condition under the flow of (2).

In the following, we recall a particular robustness notion for system (1) denoted as practical asymptotic gain (pAG) property [2].

Definition 2.5 (pAG). System (1) is said to satisfy the practical asymptotic gain (pAG) property if there exists a class $-\mathcal{K}_{\infty}$ function $\eta$ and $q \geq 0$ such that, for all $w \in M$ and all inputs $d(\cdot)$, solutions are defined for all $t \geq 0$ and the following holds:

$$
\limsup _{t \rightarrow+\infty}|W(t, w ; d)|_{\mathcal{W}_{w}} \leq \eta(\|d\|)+q
$$

If $q=0$, then we say that the asymptotic gain (AG) property holds. If (3) holds with $q=0$ and $\|d\|=0$, we say that the systems (1) and (2) satisfy the global zero-attractivity (0-GATT) property. 
The generalized notion of ISS for multistable systems in [2] replaces the Lyapunov stability requirement with Assumption 2.4 and is formalized as follows.

Definition 2.6. System (6) is said to be ISS with respect to the input $d$ and the invariant set $\mathcal{W}$ if and only if $\mathcal{W}$ satisfies Assumption 2.4 and (6) has the AG property.

We will then consider a characterization of the ISS property in Definition 2.6 in terms of a Lyapunov dissipation inequality.

Definition 2.7 (ISS-Lyapunov function). $A \mathcal{C}^{1}$ function $V: M \rightarrow \mathbb{R}$ is a practical ISS-Lyapunov function for (6) if there exists $\mathcal{K}_{\infty}$ functions $\alpha_{1}, \alpha, \gamma$ and $q \geq 0$ such that, for all $w \in M$ and all $d \in D$, the following holds:

$$
\begin{aligned}
& \alpha_{1}\left(|w|_{\mathcal{W}_{w}}\right) \leq V(w) \\
& D V(w) F(w, d) \leq-\alpha\left(|w|_{\mathcal{W}_{w}}\right)+\gamma(|d|)+q .
\end{aligned}
$$

If (5) holds with $q=0$, then $V$ is said to be an ISSLyapunov function. If, in addition, $V\left(\mathcal{W}_{w, i}\right)$ is a singleton for all $i \in\{1,2, \ldots, K\}$, then $V$ is said to be an ISSLyapunov function constant on invariant sets. If, furthermore, $D V\left(\mathcal{W}_{w}\right)=0$, then $V$ is said to be an ISS-Lyapunov function flat on invariant sets.

Theorem 2.8 (Characterizations of ISS [2]). Consider a nonlinear system as in (1) and let $\mathcal{W}$ be an $\mathcal{W}$-limit set. System (6) is ISS with respect to input d and set $\mathcal{W}$ if and on if it admits a smooth ISS Lyapunov function constant on invariant sets. Furthermore, if system (6) satisfies Assumption 2.4, then the following facts are equivalent:

1. the system satisfies the $A G$ property;

2. the system satisfies the $p A G$ property;

3. the system admits a smooth ISS Lyapunov function flat on invariant sets;

4. the system admits a practical ISS Lyapunov function.

\subsection{Cascades}

Let $M_{x}$ and $M_{z}$ be two connected Riemannian manifolds without boundary and having dimension $n_{x}$ and $n_{z}$ respectively. Let $D$ be a closed subset of $\mathbb{R}^{m}$ containing the origin. The subject of our study is the cascade system:

$$
\begin{aligned}
& \dot{x}(t)=g(x(t), d(t)) \\
& \dot{z}(t)=f(z(t), x(t)),
\end{aligned}
$$

where $g(x, d): M_{x} \times D \rightarrow T_{x} M_{x}$ and $f(z, x): M_{z} \times$ $M_{x} \rightarrow T_{z} M_{z}$ are two Lipschitz continuous mappings, and $d(\cdot)$ is any locally essentially bounded and measurable input signal taking values in $D$. We denote by $X(t, x ; d)$ the uniquely defined solution of (6a) at time $t$ fulfilling $X(0, x ; d)=x$, under the input $d(\cdot)$. In a similar way, we denote by $Z(t, z ; X)$ the uniquely defined solution of $(6 \mathrm{~b})$ at time $t$ fulfilling $Z(0, z ; X)=z$, under the input $X(\cdot)$. Finally, we denote by $y=(x, z) \in M_{x} \times M_{z}$ the joint state and by $Y(t, y ; d)$ the uniquely defined solution of $(6)$ at time $t$ fulfilling $Y(0, y ; d)=y$ under the input $d(\cdot)$.

We also consider the unperturbed cascade system:

$$
\begin{aligned}
& \dot{x}(t)=g(x(t), 0) \\
& \dot{z}(t)=f(z(t), x(t)) .
\end{aligned}
$$

\section{Multistability of the driving system}

In this Section, we derive sufficient conditions for the ISS stability of the cascade system (6), when assuming the multistable behavior of the driving system (7a).

Let $\mathcal{W}_{x}$ denote a $\mathcal{W}$-limit set of the driving system (7a). The set $\mathcal{W}_{x}$ is assumed to satisfy the following

Assumption 1. (Multistability without cycles of driving system) The driving system (7a) satisfies the no-cycle condition and, moreover, each atom of the decomposition of $\mathcal{W}_{x}$ is a singleton, namely

$$
\mathcal{W}_{x, i}=\left\{x_{i}\right\} \quad \text { with } x_{i} \in M_{x} \text { for all } i=1, \ldots, K .
$$

We are looking for a $\mathcal{W}$-limit set for $(7)$ which satisfies the no-cycle condition under the flow of the cascade system (7). In order to characterize such a set in terms of its finest decomposition, we could assume that all trajectories $X(t, x ; 0)$ are globally attracted to one of the $\omega$-limit sets in $\mathcal{W}_{x}$, i.e. for all $x \in M_{x}$ there exists $i \in\{1, \ldots, K\}$ such that $\lim _{t \rightarrow+\infty} X(t, x ; 0)=x_{i}$. Then, for any fixed trajectory $X(t, x ; 0)$ converging to some $x_{i}$, we may view the differential system $(7 \mathrm{~b})$ as the time-varying system:

$$
\dot{z}(t)=F(t, z(t)):=f(z(t), X(t, x ; 0)),
$$

and subsequently define the autonomous time-invariant systems:

$$
\dot{z}(t)=F_{i}(z(t)):=f\left(z(t), x_{i}\right), i=1, \ldots, K,
$$

with the property that $F(t, z) \rightarrow F_{i}(z)$ as $t \rightarrow+\infty$. locally uniformly in $z \in M_{z}$ for some $i=1, \ldots, K$. Equation (8) is called asymptotically autonomous with limit equation (9). Let $\mathcal{W}_{z}^{(i)}$ denote a $\mathcal{W}$-limit set of the limit equation (9) for $i=1, \ldots, K$. Then, a natural question that arises from this setting is whether the sought $\mathcal{W}$-limit set for $(7)$ can be selected as:

$$
\mathcal{W}_{\Theta}:=\bigcup_{i=1}^{K}\left(\left\{x_{i}\right\} \times \mathcal{W}_{z}^{(i)}\right)
$$

In order to answer the question, we recall the following result due to Thieme [16, Corollary 4.3]: if the limit equation (9) satisfies the no-cycle condition and the asymptotically autonomous system (8) verifies boundedness of trajectories, then the $\omega$-limit sets of (8) are subsets of the compact invariant subsets of (9). For this reason, we make the following 
Assumption 2. (No-cycle condition of each limit equations) For all $i=1, \ldots, K$, each limit equation (9), thus each set $\mathcal{W}_{z}^{(i)}$, satisfies the no-cycle condition.

We are now ready to state the main result of this section.

Theorem 3.1. Let Assumptions 1 and 2, hold. If:

- the driving system (6a) is ISS wrt input d and set $\mathcal{W}_{x}$

- the driven system (6b) is ISS wrt input $|x|^{1}$ and one of the sets $\mathcal{W}_{z}^{(i)}$,

then:

- $\mathcal{W}_{\Theta}$ qualifies as a $\mathcal{W}$-limit set for system (7) and admits a finite decomposition without cycles under the flow of (7);

- the cascade system (6) is ISS wrt input d and the set $\mathcal{W}_{\Theta}$.

In particular, a filtration ordering for $\mathcal{W}_{\Theta}$ is inherited by the filtration orderings of $\mathcal{W}_{x}$ and $\mathcal{W}_{z}^{(i)}$ as follows:

$$
\mathcal{W}_{z, h}^{(i)} \prec \mathcal{W}_{z, k}^{(i)} \Leftrightarrow\left\{x_{i}\right\} \times \mathcal{W}_{z, h}^{(i)} \prec\left\{x_{i}\right\} \times \mathcal{W}_{z, k}^{(i)},
$$

for all $i \in\{1, \ldots, K\}$ and all $h, k \in\left\{1, \ldots, K_{i}\right\}$.

Proof. Step 1: $\mathcal{W}_{\Theta}$ is a $\mathcal{W}$-limit set. We are now going to prove that $\mathcal{W}_{\Theta}$ qualifies itself as a $\mathcal{W}$-limit set for system (7). The reader is referred to [16] for the forthcoming notions of semiflow, $\omega$-limit set, $\omega-\Theta_{i}$-limit set, limit semiflow, asymptotically autonomous semiflow, isolated compact $\Phi_{i^{-}}$and $\Theta_{i^{-}}$-invariant subsets, precompact $\Phi_{i}$-orbit. Let $\Phi_{i}$ and $\Theta_{i}$ respectively denote the asymptotically autonomous semiflow and the limit semiflow associated with (8) and (9) for $i=1, \ldots, K$. Consider the following four facts: 1) since ISS holds for (7b), any $\Theta$ orbit is pre-compact; 2) by Assumption 2, the $\omega$ - $\Theta_{i}$-limit sets are isolated compact $\Theta_{i}$-invariant subsets of $M_{z} ; 3$ ) condition (E) in [16, Section 4] is satisfied by Assumption 2 ; 4) by virtue of Lemma Appendix A.1 and in particular of estimate (A.11), any forward $\Phi$-orbit is pre-compact. Therefore, by virtue of Corollary 4.3 and Remark 4.4 in [16], it is concluded that any $\Phi_{i}$-orbit converges towards a compact $\Theta_{i}$-invariant subset of $M_{x}$, namely that

$$
\omega \text { - } \Phi_{i} \text {-limit sets } \subseteq \omega \text { - } \Theta_{i} \text {-limit sets, } i=1, \ldots, K .
$$

By using the same arguments on the reverse semiflows, it is also concluded that

$$
\alpha \text { - } \Phi_{i} \text {-limit sets } \subseteq \alpha \text { - } \Theta_{i} \text {-limit sets, } i=1, \ldots, K .
$$

The joint contribution of (12) and (13) yields the result that all $\alpha$ - and $\omega$-limit sets of (8) are contained in $\mathcal{W}_{z}^{(i)}$ and this set is therefore compact, invariant, and globally attractive under the flow (8). By iterating the latter result for all $i=1, \ldots, K$, it follows that any $\alpha$ - and $\omega$-limit set of the cascade system (7) is contained in $\mathcal{W}_{\Theta}$, which is compact, invariant, and globally attractive under the flow (7). Therefore $\mathcal{W}_{\Theta}$ qualifies itself as a $\mathcal{W}$-limit set for system (7).

Step 2: no-cycle condition for $\mathcal{W}_{\Theta}$. Second, we prove that $\mathcal{W}_{\Theta}$ admits a finite decomposition without cycles under the flow of (7). Let $\mathcal{W}_{z, h}^{(i)}$ denote the $h$-th atom of the decomposition of $\mathcal{W}_{z}^{(i)}$, for $i=1, \ldots, K$ and $h=1, \ldots K_{i}$.

We prove statement (11) as follows. To see the forward direction of the implication pick $i=1, \ldots, K$ and $k, h=$ $1, \ldots, K_{i}$ such that $\mathcal{W}_{z, h}^{(i)} \prec \mathcal{W}_{z, k}^{(i)}$ holds. We would like to show that there exists $y_{a} \in M_{x} \times M_{z}$ such that, under the flow of (7), $\alpha\left(y_{a}\right) \subseteq\left\{x_{i}\right\} \times \mathcal{W}_{z, k}^{(i)}$ and $\omega\left(y_{a}\right) \subseteq\left\{x_{i}\right\} \times \mathcal{W}_{z, h}^{(i)}$, and hence $\mathcal{A}\left(\left\{x_{i}\right\} \times \mathcal{W}_{z, h}^{(i)}\right) \cap \mathcal{R}\left(\left\{x_{i}\right\} \times \mathcal{W}_{z, k}^{(i)}\right)$ has at least one element $y_{a}$. By definition, $\mathcal{W}_{z, h}^{(i)} \prec \mathcal{W}_{z, k}^{(i)}$ implies the existence of some $z_{a} \in M_{z}$ such that, under the flow of (9), $\alpha\left(z_{a}\right) \subseteq \mathcal{W}_{z, k}^{(i)}$ and $\omega\left(z_{a}\right) \subseteq \mathcal{W}_{z, h}^{(i)}$. Consider the constant trajectory $X\left(t, x_{i} ; 0\right) \equiv x_{i}$ for all $t \in \mathbb{R}$. For this trajectory the flow of (9) exactly matches the flow of (7) in the $z$ coordinates. We can then select $y_{a}=\left(x_{i}, z_{a}\right)$ as the point for which (11) is satisfied. For the converse implication pick $y_{a} \in M_{x} \times M_{z}$, and $h, k \in\left\{1, \ldots, K_{i}\right\}$ with $h \neq k$, such that $\alpha\left(y_{a}\right) \subseteq\left\{x_{i}\right\} \times \mathcal{W}_{z, k}^{(i)}$ and $\omega\left(y_{a}\right) \subseteq\left\{x_{i}\right\} \times \mathcal{W}_{z, h}^{(i)}$. Let $x_{a}$ and $z_{a}$ respectively denote the $x$ and $z$ components of $y_{a}$. Since $\alpha\left(x_{a}\right)=\omega\left(x_{a}\right)=\left\{x_{i}\right\}$, we can recall that Assumption 1 rules out the existence of 1-cycles among the atoms of $(7 \mathrm{a})$, therefore it holds that $x_{a}=x_{i}$ and, moreover, $X\left(t, x_{a} ; 0\right) \equiv x_{i}$ for all $t \in \mathbb{R}$. The latter statement implies that the flow of $Z\left(t, z_{a} ; X(\cdot)\right)$ is given by limit equation (9) for all $t \in \mathbb{R}$, and hence conclude that, under the flow of (9), we have $\alpha\left(z_{a}\right) \subseteq \mathcal{W}_{z, k}^{(i)}$ and $\alpha\left(z_{a}\right) \subseteq \mathcal{W}_{z, k}^{(j)}$, thus satisfying $\mathcal{W}_{z, k}^{(i)} \prec \mathcal{W}_{z, k}^{(j)}$.

We are now going to rule out the existence of a 1cycle around each atom $\left\{x_{i}\right\} \times \mathcal{W}_{z, h}^{(i)}$. By contradiction, assume that there exists a couple of indices $i, h$ such that $\left[\mathcal{R}\left(\left\{x_{i}\right\} \times \mathcal{W}_{z, h}^{(i)}\right) \cap \mathcal{A}\left(\left\{x_{i}\right\} \times \mathcal{W}_{z, h}^{(i)}\right)\right] \backslash\left(\left\{x_{i}\right\} \times \mathcal{W}_{z, h}^{(i)}\right) \neq$ $\emptyset$. This implies the existence of $y_{a}=\left(x_{a}, z_{a}\right) \notin\left\{x_{i}\right\} \times \mathcal{W}_{z, h}^{(i)}$ such that, under the flow of $(7), \alpha\left(y_{a}\right) \cap \omega\left(y_{a}\right) \subseteq\left\{x_{i}\right\}^{z, h}$ $\mathcal{W}_{z, h}^{(i)}$. Two cases then arise:

- $x_{a} \neq x_{i}$. Given that $\alpha\left(x_{a}\right)=\omega\left(x_{a}\right)=\left\{x_{i}\right\}$ and given the independence of the flow of (7a) from the flow of (7), the latter statement contradicts Assumption 1;

- $x_{a}=x_{i}$ and $z_{a} \notin \mathcal{W}_{z, h}^{(i)}$, which implies $X\left(t, x_{a} ; 0\right) \equiv$ $x_{i}$ for all $t \in \mathbb{R}$, thus making the flow of (8) exactly match the flow of $(9)$ in the $z$ coordinates. Note that $\alpha\left(z_{a}\right) \cap \omega\left(z_{a}\right) \subseteq \mathcal{W}_{z, h}^{(i)}$ with $z_{a} \notin \mathcal{W}_{z, h}^{(i)}$, thus contradicting Assumption 2.

We are now going to rule out the existence of a $r$-cycle among the atoms of the decomposition (10). By contradiction, assume that a $r$-cycle exists. Two cases arise: 1) 
all atoms of the $r$-cycle have the same $x$ component, say $x_{i} ; 2$ ) at least one atom of the $r$-cycle has a different $x$ component. Case 1) implies the existence of a sequence

$$
\left\{x_{i}\right\} \times \mathcal{W}_{z, k_{1}}^{(i)} \prec \cdots \prec\left\{x_{i}\right\} \times \mathcal{W}_{z, k_{r}}^{(i)} \prec\left\{x_{i}\right\} \times \mathcal{W}_{z, k_{1}}^{(i)}
$$

with $k_{1}, \ldots, k_{r} \in\left\{1, \ldots, K_{i}\right\}$. By equivalence (11), sequence (14) implies the existence of a sequence $\mathcal{W}_{z, k_{1}}^{(i)} \prec$ $\ldots \prec \mathcal{W}_{z, k_{r}}^{(i)} \prec \mathcal{W}_{z, k_{1}}^{(i)}$, which in turn contradicts Assumption 2. Case 2) implies the existence of a sequence

$$
\begin{aligned}
& \left\{x_{i_{1}}\right\} \times \mathcal{W}_{z, k_{1,1}}^{\left(i_{1}\right)} \prec \cdots \prec\left\{x_{i_{1}}\right\} \times \mathcal{W}_{z, k_{1, m_{1}}}^{\left(i_{1}\right)} \prec \\
& \left\{x_{i_{2}}\right\} \times \mathcal{W}_{z, k_{2,1}}^{\left(i_{2}\right)} \prec \cdots \prec\left\{x_{i_{2}}\right\} \times \mathcal{W}_{z, k_{2, m_{2}}}^{\left(i_{2}\right)} \prec \\
& \cdots \prec\left\{x_{i_{r}}\right\} \times \mathcal{W}_{z, k_{r, m_{r}}}^{\left(i_{r}\right)} \prec\left\{x_{i_{1}}\right\} \times \mathcal{W}_{z, k_{1,1}}^{\left(i_{1}\right)},
\end{aligned}
$$

with $i_{1}, \ldots, i_{r} \in\{1, \ldots, K\}$, where at least one index does not equal the others. Without loss of generality, select $i_{r}$ as such index. Then, sequence (15) implies the existence of a sequence $x_{a_{1}}, \ldots, x_{a_{r}}$ such that, under the flow of (7a), it holds:

$$
\begin{aligned}
& \alpha\left(x_{a_{l}}\right)=\left\{x_{i_{l+1}}\right\}, \omega\left(x_{a_{l}}\right)=\left\{x_{i_{l}}\right\} \text { for } l \in\{1, \ldots, r-1\} \\
& \alpha\left(x_{a_{r}}\right)=\left\{x_{i_{1}}\right\}, \omega\left(x_{a_{r}}\right)=\left\{x_{i_{r}}\right\} .
\end{aligned}
$$

We can then pick up a subsequence of $x_{a_{1}}, \ldots, x_{a_{r}}$, say $x_{b_{1}}, \ldots, x_{b_{\tilde{r}}}$ such that $x_{b_{n}} \neq x_{b_{n+1}}$ for all $n \in\{1, \ldots, \tilde{r}\}$, $\tilde{r}>1$. This implies the existence of a $\tilde{r}$-cycle under the flow of (7a) which represents a contradiction with Assumption 1 .

Step 3: ISS of the cascade system. Finally, we prove ISS of the cascaded system (6). Indeed, by virtue of Lemma Appendix A.1, we can write the asymptotic estimate (A.5) with respect to an arbitrary compact set $\tilde{\mathcal{W}} \subset M_{x} \times M_{z}$. Specifically, we can write (A.5) with respect to the set $\mathcal{W}_{\Theta}$. Since $\mathcal{W}_{\Theta}$ satisfies the no-cycle condition under the flow of (7), estimate (A.5) represents the practical asymptotic gain (pAG) property as introduced in [2]. By virtue of Theorem 2 in [2], we conclude ISS stability of (6) with respect to input $d$ and $\mathcal{W}_{\Theta}$.

\section{Multi-almost periodicity + incremental ISS}

In this Section, we derive sufficient conditions for the ISS stability of the cascade system (6), based on the following two key assumptions:

- the asymptotic behavior of the driving system (7a) is characterized by fixed points, periodic orbits, and almost-periodic attractors;

\footnotetext{
${ }^{1}$ In the definition of $|x|$ for $x \in M_{x}$ we use the following result. For a Riemannian manifold $M_{x}$, the Euclidean metric is uniformly equivalent to the Riemannian metric, i.e. there exists $\nu_{1}, \nu_{2} \in \mathcal{K}_{\infty}$ such that $\nu_{1}\left(\mathfrak{d}\left(x, O_{x}\right)\right) \leq|x| \leq \nu_{2}\left(\mathfrak{d}\left(x, O_{x}\right)\right)$, with $O_{x}$ denoting the "origin" element of $M_{x}$. Therefore, we define $|x|$ as the Euclidean norm if $M_{x}$ is Euclidean, and $|x|:=\mathfrak{d}\left(x, O_{x}\right)$ if $M_{x}$ is not Euclidean, thus implicitly making use of $\nu_{1}, \nu_{2}$ in all subsequent proofs.
}

- the driven system is incremental ISS (in the sense of $[1])$.

In particular, we are mainly concerned with the response of an incremental ISS system under different type of forcing signals: asymptotically constant, asymptotically periodic, and asymptotically almost periodic. To the aim of characterizing the class of input signal in consideration, we use the following two definitions.

Definition 4.1. An $\mathbb{R}^{n}$-valued function $v$ defined on $(-\infty$, $+\infty)$ is said to be almost periodic [4] if it is continuous on $(-\infty,+\infty)$ and if for each $\epsilon>0$ there exists a real number $l>0$ such that every intervals in $(-\infty,+\infty)$ of length $l$ contains $\tau$ such that

$$
|v(t+\tau)-v(t)|<\epsilon
$$

holds for all $t \in(-\infty,+\infty)$. For all $\epsilon$, each $\tau$ in every interval in $(-\infty,+\infty)$ of length $l$ will be called a $\epsilon-$ displacement.

Definition 4.2. An $\mathbb{R}^{n}$-valued function $u$ defined on $[0,+\infty)$ is said to be asymptotically almost periodic if it can be decomposed as the sum $u=u_{1}+u_{2}$, where $u_{1}$ is the restriction to $[0,+\infty)$ of an almost periodic function, and $u_{2}$ is a continuous function defined on $[0,+\infty)$ such that $\lim _{t \rightarrow+\infty} u_{2}(t)=0$ as $t \rightarrow+\infty$.

An almost-periodic attractor $\Upsilon_{x, i}, i=1, \ldots, N_{a p}$ of the driving system (7a) will denote a compact invariant subset defined as

$$
\Upsilon_{x, i}:=\operatorname{clos}\{X(t, x ; 0), t \in \mathbb{R}\}
$$

for some $x \in \Upsilon_{x, i}$, and where $X(\cdot, x ; 0)$ is an almost periodic function and is neither a periodic function nor a fixed point. In virtue of [3, Theorem 6.7], every motion with initial condition in $\Upsilon_{x, i}$ is almost periodic with the same set of $\epsilon$-displacements.

A periodic orbit $\Gamma_{x, j}, j=1, \ldots, N_{p}$ of the driving system (7a) will denote a compact invariant subset satisfying the following property:

$$
\begin{aligned}
& \forall i \in\left\{1, \ldots, N_{p}\right\} \quad \exists T_{i}>0 \quad \text { such that } \\
& X\left(t+T_{i}, x ; 0\right)=X(t, x ; 0) \quad \forall x \in \Gamma_{x, i} \quad \forall t \in \mathbb{R} .
\end{aligned}
$$

Note that, since a periodic orbit is a one-dimensional closed curve, any point on a periodic orbit can be characterized by a scalar phase that uniquely determines its position on the periodic orbit. For $i=1, \ldots, N_{p}$, let $P_{i}: \Gamma_{x, i} \rightarrow[0,2 \pi)$ denote the smooth bijective phase map associating to each point $x$ on the orbit $\Gamma_{x, i}$, its phase $\theta \in[0,2 \pi)$. Note that the map $P$ can be defined in such a way that, along a trajectory starting on the limit cycle, $\theta$ evolves linearly in time, namely the following property holds whenever $x \in \Gamma_{x, i}$ :

$$
P_{i}(X(t, x ; 0))=\left(\left(\frac{2 \pi}{T_{i}} t\right)+P_{i}(x)\right) \bmod 2 \pi .
$$


with $i=1, \ldots, N_{p}$. We can then define:

$$
X^{(i)}(t):=X\left(t, P_{i}^{-1}(0) ; 0\right), i=1, \ldots, N_{p} .
$$

Note that $X^{(i)}(t)$ is periodic with period $T_{i}$ and, in virtue of (17), it holds for all $i=1, \ldots, N_{p}$, all $t \in \mathbb{R}$, and for all $\theta \in[0,2 \pi)$, that:

$$
X^{(i)}\left(t+\frac{\theta}{2 \pi} T_{i}\right)=X\left(t, P_{i}^{-1}(\theta) ; 0\right) .
$$

Let now $\mathcal{W}_{x}$ denote a $\mathcal{W}$-limit set of the driving system (7a). The set $\mathcal{W}_{x}$ is assumed to satisfy the following:

Assumption 3. (Multi-almost periodicity without cycles of driving system) The driving system (7a) satisfies the nocycle condition and, moreover, the decomposition of $\mathcal{W}_{x}$ reads as the disjoint union of the following sets:

$$
\bigcup_{k=1}^{N_{a p}+N_{p}+N_{f}} \mathcal{W}_{x, k}=\bigcup_{i=1}^{N_{a p}} \Upsilon_{x, i}+\bigcup_{j=1}^{N_{p}} \Gamma_{x, j}+\bigcup_{h=1}^{N_{f}}\left\{x_{h}\right\}
$$

where $\Upsilon_{x, i} \subset M_{x}$ for $i=1, \ldots, N_{a p}$ is an almost periodic attractor, $\Gamma_{x, j} \subset M_{x}$ for $j=1, \ldots, N_{p}$ is a periodic orbit, and $x_{h} \in M_{x}$ for $h=1, \ldots, N_{f}$ is an isolated fixed point.

The following key assumption is made on the driven system.

Assumption 4. (Incremental ISS of driving system) System (7b) is incrementally input-to-state stable ( $\delta I S S)$ in the sense of [1]. The definition implies that the state manifold is the Euclidean space $\left(M_{z}=\mathbb{E}^{n_{z}}\right)$ and that there exists a $\mathcal{K} \mathcal{L}$ function $\beta$ and $\gamma \in \mathcal{K}_{\infty}$ such that for any $t \geq 0$, any $z_{1}, z_{2} \in \mathbb{R}^{n_{z}}$ and any couple of input $X_{1}(\cdot), X_{2}(\cdot)$, the following is true:

$$
\begin{aligned}
& \left|Z\left(t, z_{1} ; X_{1}\right)-Z\left(t, z_{2} ; X_{2}\right)\right| \leq \\
& \quad \beta\left(\left|z_{1}-z_{2}\right|, t\right)+\gamma\left(\mathfrak{d}_{[0,+\infty)}\left(X_{1}(\cdot), X_{2}(\cdot)\right)\right) .
\end{aligned}
$$

Without loss of generality, we will assume $f\left(0, O_{x}\right)=0$, where $O_{x}$ denotes the "origin" element of $M_{x}$.

We are looking for a $\mathcal{W}$-limit set of the cascade system (7), say $\mathcal{W}_{\Phi}$, which satisfies the no-cycle condition under the flow of (7). In order to provide a characterization of the set $\mathcal{W}_{\Phi}$ in terms of its finest decomposition, we first consider the case of isolated equilibria in the driving system (7a), as in the following

Claim 4.1. If $\lim _{t \rightarrow+\infty} \mathfrak{d}\left(X(t, x ; 0), x_{i}\right)=0$ for some $x_{i}$ with $i=1, \ldots, N_{f}$ as in the decomposition (19), then there exists a unique equlibrium point $z_{i} \in M_{z}$ such that:

$$
\lim _{t \rightarrow+\infty}\left|Z(t, z ; X(\cdot))-z_{i}\right|=0 .
$$

Proof. This claim is proved by a straightforward application of [1, Proposition 4.2] and [1, Proposition 4.6] for each constant input signals $x_{i}$.
We consider now the case of periodic orbits in the driving system (7a). First, we recall the following property characterizing the behavior of solutions attracted to a periodic orbit.

Definition 4.3. Periodic orbit $\Gamma_{x, i}$ is said to have the asymptotic phase property if, for all $x \in \mathcal{A}\left(\Gamma_{x, i}\right)$, there exists $\theta \in[0,2 \pi)$ such that the following limit holds true:

$$
\lim _{t \rightarrow+\infty} \mathfrak{d}\left(X(t, x ; 0), X^{(i)}\left(t+\frac{\theta}{2 \pi} T_{i}\right)\right)=0,
$$

Typically, the asymptotic phase property does not necessarily hold for subsystem (7a) and must be assumed. Second, we recall the following additional property: by virtue of [1, Proposition 4.4], the system (7b), forced with a periodic input $X^{(i)}(t)$, has a state response which asymptotically tends to a periodic function of the same period and, moreover, there exists initial condition $z^{(i), 0}$ such that $Z\left(t, z^{(i), 0}, X^{(i)}(t)\right)$ is periodic. We claim that, for a $\delta$ ISS system, if the forcing input asymptotically tends to a periodic function with phase shift $\theta \in[0,2 \pi)$, the state response also tends to a periodic function with phase shift $\theta$, namely the shape of the state response does not depend upon the phase of the input nor the initial state. We formalize this result in the following

Claim 4.2. Consider the periodic input $X^{(i)}(t)$, with pe$\operatorname{riod} T_{i}$, for some $i=1, \ldots, N_{p}$. Let $z^{(i), 0}$ be the initial condition as in [1, Proposition 4.4] such that

$$
Z^{(i)}(t):=Z\left(t, z^{(i), 0}, X^{(i)}(\cdot)\right)
$$

is the periodic state response of subsystem (7b) to input $X^{(i)}(t)$. Let $x \in M_{x}$ and assume that limit (21) holds for some $\theta \in[0,2 \pi)$. Then, it holds for all $z \in M_{z}$ that:

$$
\lim _{t \rightarrow+\infty}\left|Z(t, z ; X(\cdot, x ; 0))-Z^{(i)}\left(t+\frac{\theta}{2 \pi} T_{i}\right)\right|=0 .
$$

Proof. We first prove that a periodic input signal with nonzero phase yields a state response which asymptotically tracks a periodic function with the same phase, namely we prove that:

$$
\lim _{t \rightarrow+\infty}\left|Z\left(t, z ; X^{(i)}\left(\cdot+\frac{\theta}{2 \pi} T_{i}\right)\right)-Z^{(i)}\left(t+\frac{\theta}{2 \pi} T_{i}\right)\right|=0,
$$

for any $z \in M_{z}$ and for any $\theta \in[0,2 \pi)$. To this end, we define:

$$
\bar{z}:=Z\left(-\frac{\theta}{2 \pi} T_{i}, z, X^{(i)}\left(\cdot+\frac{\theta}{2 \pi} T_{i}\right)\right) .
$$

Note that existence of $\bar{z}$ follows from (7b) being incrementally ISS, hence complete. By using the following invariance property for non-autonomous flows:

$$
Z(t+\alpha, Z(\beta, z, X(\cdot-\beta)), X(\cdot))=Z(t+\alpha+\beta, z, X(\cdot-\beta)),
$$


with $\alpha=-\beta=\frac{\theta}{2 \pi} T_{i}$, we obtain:

$$
\begin{aligned}
& Z\left(t+\frac{\theta}{2 \pi} T_{i}, Z\left(-\frac{\theta}{2 \pi} T_{i}, z, X^{(i)}\left(\cdot+\frac{\theta}{2 \pi} T_{i}\right)\right), X^{(i)}(\cdot)\right) \\
& =Z\left(t, z, X^{(i)}\left(\cdot+\frac{\theta}{2 \pi} T_{i}\right)\right) .
\end{aligned}
$$

By using (25), equation (27) is immediately rewritten as:

$$
Z\left(t+\frac{\theta}{2 \pi} T_{i}, \bar{z}, X^{(i)}(\cdot)\right)=Z\left(t, z, X^{(i)}\left(\cdot+\frac{\theta}{2 \pi} T_{i}\right)\right) .
$$

By virtue of the incremental ISS property, it then follows from (28) that:

$$
\begin{aligned}
& \left|Z\left(t, z, X^{(i)}\left(\cdot+\frac{\theta}{2 \pi} T_{i}\right)\right)-Z\left(t+\frac{\theta T_{i}}{2 \pi}, z^{(i), 0}, X^{(i)}(\cdot)\right)\right| \\
& \leq \beta\left(\left|\bar{z}-z^{(i), 0}\right|, t+\frac{\theta}{2 \pi} T_{i}\right),
\end{aligned}
$$

By taking the limit for $t \rightarrow+\infty$, inequality (29) yields the sought result (24). We are now going to prove (23). Indeed, by [1, Proposition 4.5], it holds that:

$$
\lim _{t \rightarrow+\infty}\left|Z\left(t+\frac{\theta T_{i}}{2 \pi}, \bar{z}, X^{(i)}(\cdot)\right)-Z\left(t+\frac{\theta T_{i}}{2 \pi}, \bar{z}, X(t, x ; 0)\right)\right|
$$

equals zero for all $\theta \in[0,2 \pi)$ and all $\bar{z} \in M_{z}$ as given in definition (25). The claim is then proven by applying the triangle inequality on the arguments of limits (24) and (30).

Finally, we consider the case of almost-periodic attractors in the driving system (7a). For all $i=1, \ldots, N_{a p}$, we select an initial condition $\bar{x} \in \Upsilon_{x, i}$ and then we define the almost-periodic function $X^{[i]}(t, \bar{x}):=X(t, \bar{x} ; 0)$ for all $t \in \mathbb{R}$. Incremental stability in a compact set implies uniform convergence [9, Theorem 11], and therefore there exists a unique solution $Z^{[i]}(t, \bar{x})$ of the non-autonomous system $\dot{z}(t)=f\left(z(t), X^{[i]}(t, \bar{x})\right)$ in $M_{z}$ defined and bounded for all $t \in \mathbb{R}$ (see [9, Definition 1$]$ ), and only depending upon $\bar{x}$. We recall the following property which typically needs to be assumed for an almost-periodic attractor.

Definition 4.4. Almost-periodic attractor $\Upsilon_{x, i}$ is said to have the asymptotic almost-phase property if, for all $x \in$ $\mathcal{A}\left(\Upsilon_{x, i}\right)$, there exists $T \in \mathbb{R}$ such that the following limit holds true:

$$
\lim _{t \rightarrow+\infty} \mathfrak{d}\left(X(t, x ; 0), X^{[i]}(t+T, \bar{x})\right)=0,
$$

The following Claim and its proof are largely inspired by the works [7] and [11]. Instrumental in the proof of the Claim would be the following

Lemma 4.5. An $\mathbb{R}^{n}$-valued function $v$ defined on $[0,+\infty)$ is asymptotically almost periodic if and only if for each $\epsilon>0$ there are $l>0$ and $\alpha>0$ such that every interval in $[0,+\infty)$ of length $l$ has a number $\tau$ for which

$$
|v(t)-v(t+\tau)|<\epsilon,
$$

for all $t \geq \alpha$. Furthermore, condition (31) is equivalently rewritten as

$$
\|v(\cdot+\alpha)-v(\cdot+\alpha+\tau)\|<\epsilon
$$

where $\|\cdot\|$ denotes the standard $\infty$-norm, i.e.

$$
\|s(\cdot)\|=\sup _{t \geq 0}|s(t)| .
$$

Claim 4.3. The solution of an incrementally ISS system under asymptotically almost periodic forcing is asymptotically almost periodic. Moreover, if the input is asymptotically almost periodic and converging to $X^{[i]}(t, \bar{x})$ for some $\bar{x} \in \Upsilon_{x, i}$, then the state is converging to the unique solution $Z^{[i]}(t, \bar{x})$.

Proof. Consider the incremental ISS system (7b) and functions $\beta \in \mathcal{K} \mathcal{L}$ and $\gamma \in \mathcal{K}_{\infty}$ as in $(20)$. Let $X(\cdot)=X(t, x ; 0)$ be an asymptotically almost periodic input signal corresponding to the initial condition $x \in M_{x}$. We want to prove that, for all $z \in M_{z}$, the solution $Z(t, z ; X(\cdot))$ is asymptotically almost periodic. By Lemma 4.5, we can formulate such a goal as follows: we want to prove that, for all $z \in M_{z}$ and all $\rho>0$, there exists two constants $l^{\prime}, \alpha^{\prime}>0$ such that every interval $I \subset[0,+\infty)$ of length $l^{\prime}$ has a number $\tau^{\prime}$ for which

$$
|Z(t, z ; X(\cdot))-Z(t+\tau, z ; X(\cdot))|<\rho,
$$

for all $t \geq \alpha^{\prime}$. Condition (33) can be equivalently rewritten as:

$$
\left|Z\left(t+\alpha^{\prime}, z ; X(\cdot)\right)-Z\left(t+\alpha^{\prime}+\tau, z ; X(\cdot)\right)\right|<\rho,
$$

for all $t \geq 0$. To this end, pick $z \in M_{z}$ and $\rho>0$. Since $X(\cdot)$ is asymptotically almost periodic, we can select $l, \alpha>$ 0 such that every interval $I \subset[0,+\infty)$ of length $l$ has a number $\tau$ for which

$$
\mathfrak{d}_{[0,+\infty)}(X(\cdot+\alpha), X(\cdot+\alpha+\tau))<\frac{\rho}{2} .
$$

Moreover, due to property (26), we observe that, for all $t \geq 0$ :

$$
\begin{aligned}
|Z(t+\alpha, z ; X(\cdot))-Z(t+\alpha+\tau, z ; X(\cdot))| & \\
= & \left|Z\left(t, z_{\alpha} ; X(\cdot+\alpha)\right)-Z\left(t+\tau, z_{\alpha} ; X(\cdot+\alpha)\right)\right| \\
= & \mid Z\left(t, z_{\alpha} ; X(\cdot+\alpha)\right) \\
& \quad-Z\left(t, Z\left(\tau, z_{\alpha} ; X(\cdot+\alpha)\right) ; X(\cdot+\alpha+\tau)\right) \mid,
\end{aligned}
$$

with $z_{\alpha}:=Z(\alpha, z ; X(\cdot))$. Therefore, due to (6) being incremental ISS, it holds:

$$
\begin{aligned}
& |Z(t+\alpha, z ; X(\cdot))-Z(t+\alpha+\tau, z ; X(\cdot))| \\
& \leq \beta\left(\left|z_{\alpha}-Z\left(\tau, z_{\alpha} ; X(\cdot+\alpha)\right)\right|, t\right) \\
& \quad+\gamma\left(\mathfrak{d}_{[0,+\infty)}(X(\cdot+\alpha)-X(\cdot+\alpha+\tau))\right)
\end{aligned}
$$


for all $t \geq 0$. Since $\beta$ is a $\mathcal{K} \mathcal{L}$ function and boundedness of trajectories holds for an asymptotically almost periodic input, we can find a time $t_{\rho / 2}$ such that

$$
\beta\left(\left|z_{\alpha}-Z\left(\tau, z_{\alpha} ; X(\cdot+\alpha)\right)\right|, t\right) \leq \frac{\rho}{2},
$$

for all $t \geq t_{\rho / 2}$. By using (35) and (38), estimate (36) reads as:

$$
|Z(t+\alpha, z ; X(\cdot))-Z(t+\alpha+\tau, z ; X(\cdot))| \leq \rho,
$$

for all $t \geq t_{\rho / 2}$. It is then clear that, for all $\rho>0$, there exists constants $l^{\prime}:=l, \alpha^{\prime}:=\alpha+t_{\rho / 2}$ such that every interval $I \subset[0,+\infty)$ of length $l^{\prime}:=l$ has a number $\tau^{\prime}:=\tau$ for which (33) holds for all $t \geq \alpha^{\prime}$, hence $Z(t, z ; X(\cdot))$ is asymptotically almost periodic. The second statement of the Claim is easily proved by [1, Proposition 4.5].

To the end of characterizing the $\mathcal{W}$-limit set for $(7)$, we provide the following definitions. Let

$$
\Upsilon_{i}=\operatorname{clos}\left\{\left(\begin{array}{c}
X^{[i]}(t) \\
Z^{[i]}(t)
\end{array}\right), t \in \mathbb{R}\right\} \text { for all } i=1, \ldots, N_{a p}
$$

as the almost-periodic attractor corresponding to $\Upsilon_{x, i}$ in the decomposition (19). Let

$$
\Gamma_{i}=\left\{\left(\begin{array}{l}
X^{(i)}(t) \\
Z^{(i)}(t)
\end{array}\right), t \in\left[0, T_{i}\right)\right\} \text { for all } i=1, \ldots, N_{p}
$$

as the periodic orbit corresponding to $\Gamma_{x, i}$ in the decomposition (19), with $X^{(i)}(t), Z^{(i)}(t)$ as in Claim 4.2. Observe that, by virtue of Claims 4.2 and 4.3 , trajectories $\left(X^{[i]}(t)\right.$ $\left.Z^{[i]}(t)\right)$ and $\left(X^{(i)}(t), Z^{(i)}(t)\right)$ are time-varying, yet sets $\Upsilon_{i}, \Gamma_{i}$ are time independent. Define

$$
y_{i}=\left(\begin{array}{c}
x_{i} \\
z_{i}
\end{array}\right) \text { for all } i=1, \ldots, N_{f}
$$

as the isolated fixed point corresponding to $\left\{x_{i}\right\}$ in the decomposition (19), with $x_{i}, z_{i}$ as in Claim 4.1.

Then, a natural question that arises from this setting is whether the sought $\mathcal{W}$-limit set for (7) can be selected as:

$$
\mathcal{W}_{\Theta}=\bigcup_{i=1}^{N_{a p}} \Upsilon_{i} \cup \bigcup_{j=1}^{N_{p}} \Gamma_{j} \cup \bigcup_{h=1}^{N_{f}}\left\{y_{h}\right\}
$$

Let:

$$
\mathcal{W}_{i}:= \begin{cases}\Upsilon_{i} & \text { for } i=1, \ldots, N_{a p} \\ \Gamma_{i-N_{a p}} & \text { for } i=N_{a p}+1, \ldots, N_{a p}+N_{p} \\ \left\{y_{i-N_{a p}-N_{p}}\right\} & \text { for } i=N_{a p}+N_{p}+1, \ldots, N_{a p}+N_{p}+N_{f} .\end{cases}
$$

Then, the set $\mathcal{W}_{\Theta}$ would read as $\mathcal{W}_{\Theta}=\bigcup_{i=1}^{N_{a p}+N_{p}+N_{f}} \mathcal{W}_{i}$.

We are now ready to state the main result of this section.

Theorem 4.6. Let Assumptions 3 and 4 hold. Assume that the asymptotic phase (respectively, almost-phase) property holds for all $\Gamma_{x, i} s$ (respectively, for all $\Upsilon_{x, i}$ ). Then, the set $\mathcal{W}_{\Theta}$ in (43) qualifies as a $\mathcal{W}$-limit set and admits a finite decomposition without cycles (in the sense of Definition 2.4) under the flow of (7). Moreover, if the driving system (6a) is ISS wrt input d and the set $\mathcal{W}_{x}$, then the cascade system (6) is ISS wrt input d and the set $\mathcal{W}_{\Theta}$. In particular, a filtration ordering for $\mathcal{W}_{\Theta}$ is inherited by the filtration orderings of $\mathcal{W}_{x}$ and $\mathcal{W}_{z}^{(i)}$ as follows:

$$
\mathcal{W}_{x, i} \prec \mathcal{W}_{x, j} \Leftrightarrow \mathcal{W}_{i} \prec \mathcal{W}_{j}
$$

for all $i, j=1, \ldots, N_{a p}+N_{p}+N_{f}$.

Proof. Step 1: $\mathcal{W}_{\Theta}$ is a $\mathcal{W}$-limit set. By virtue of Claims $4.1,4.2$, and 4.3 , all $\omega$-limit sets of (7) are contained in (43). By virtue of Claim 4.4, it will also be clear that all $\alpha$-limit sets of (7) are contained in (43).

Step 2: no-cycle condition for $\mathcal{W}_{\Theta}$. We prove that $\mathcal{W}_{\Theta}$ admits a finite decomposition without cycles under the flow of (7). To this end, we first prove statement (44) as follows. For direction $\Rightarrow$, pick $i, j=1, \ldots, N_{a p}+N_{p}+N_{f}$ such that $\mathcal{W}_{x, i} \prec \mathcal{W}_{x, j}$ holds. We would like to show that there exists $y_{a} \in M_{x} \times M_{z}$ such that, under the flow of (7), $\alpha\left(y_{a}\right)=\mathcal{W}_{j}$ and $\omega\left(y_{a}\right)=\mathcal{W}_{i}$, and hence $\mathcal{A}\left(\mathcal{W}_{j}\right) \cap \mathcal{R}\left(\mathcal{W}_{i}\right)$ has at least one element $y_{a}$. By definition, $\mathcal{W}_{x, i} \prec \mathcal{W}_{x, j}$ implies the existence of some $x_{a} \in M_{x}$ such that, under the flow of (7a), $\alpha\left(x_{a}\right)=\mathcal{W}_{x, j}$ and $\omega\left(x_{a}\right)=\mathcal{W}_{x, i}$. It follows that trajectory $\bar{X}(t):=X\left(t, x_{a} ; 0\right)$ satisfies:

$$
\begin{aligned}
& \lim _{t \rightarrow+\infty} \mathfrak{d}\left(\bar{X}(t), X_{S S}^{(i)}(t)\right)=0, \\
& \lim _{t \rightarrow-\infty} \mathfrak{d}\left(\bar{X}(t), X_{S S}^{(j)}(t)\right)=0,
\end{aligned}
$$

where, for $h=1, \ldots, N_{a p}+N_{p}+N_{f}$, the steady-state trajectory has been defined as

$$
X_{S S}^{(h)}(t):=\left\{\begin{array}{c}
X^{[h]}\left(t, x^{[h]}\right) \text { for } h=1, \ldots, N_{a p} \\
X^{\left(h-N_{a p}\right)}\left(t+\frac{\theta_{h-N_{a p}}}{2 \pi} T_{h-N_{a p}}\right) \\
\quad \text { for } h=N_{a p}+1, \ldots, N_{a p}+N_{p} \\
x_{h-N_{a p}-N_{p}} \\
\quad \text { for } h=N_{a p}+N_{p}+1, \ldots, N_{a p}+N_{p}+N_{f} .
\end{array}\right.
$$

where $x^{[h]} \in \Upsilon_{x, h}$ and $\theta_{h} \in[0,2 \pi)$ are selected in order to satisfy (45), and (46).

In the following claim, we characterize the backward attractor for the $\delta$ ISS system (7b) driven by $\bar{X}(t)$.

Claim 4.4. There exists an initial condition $z_{a} \in M_{z}$ such that:

$$
\alpha\left(\left(\begin{array}{c}
\bar{X}(t) \\
Z\left(t, z_{a} ; \bar{X}(\cdot)\right)
\end{array}\right)\right) \subseteq \mathcal{W}_{j} .
$$

Proof. Consider the non-autonomous systems

$$
\begin{aligned}
& \dot{z}(t)=F(t, z(t)):=f(z(t), \bar{X}(t)) . \\
& \dot{z}(t)=F_{S S}(t, z(t)):=f\left(z(t), X_{S S}^{(j)}(t)\right),
\end{aligned}
$$

where $F(t, z), F_{S S}(t, z)$ are defined for all $t \in \mathbb{R}$ and for all $z \in M_{z}$, due to $\bar{X}(t), X_{S S}^{(j)}(t)$ being defined for all $t \in \mathbb{R}$. In 
[9, Theorem 11] it is estabilished that incremental stability in a compact set implies uniform convergence. Specifically, the uniform convergence [9, Definition 1] implies that:

- (P1) all solutions $Z\left(t, z_{0} ; \bar{X}(\cdot-T)\right)$ of (48) and all solutions $Z\left(t, z_{0} ; X_{S S}^{(j)}(\cdot-T)\right)$ of (49) exist for all $T \in$ $\mathbb{R}$, for all $t \geq 0$, and for all initial conditions $z_{0} \in M_{z}$;

- (P2) there exists a unique solution $\bar{Z}(t)$ of (48) in $M_{z}$ defined and bounded for all $t \in \mathbb{R}$.

Select $z_{a}=\bar{Z}(0)$. Clearly, we have that $\bar{Z}(t)=Z\left(t, z_{a} ; \bar{X}(\cdot)\right)$. To the end of proving the limit $(47)$, we recall that $\delta$ ISS implies:

$$
\begin{aligned}
& \left|\bar{Z}(t)-Z\left(t, z_{0}, X_{S S}^{(j)}(\cdot)\right)\right| \leq \\
& \quad \beta\left(\left|\bar{Z}(0)-z_{0}\right|, t\right)+\gamma\left(\mathfrak{d}_{[0, t]}\left(\bar{X}(\cdot), X_{S S}^{(j)}(\cdot)\right)\right),
\end{aligned}
$$

for all $z_{0} \in M_{z}$ and for all $t \geq 0$. Note that the $\delta$ ISS inequality (50) holds with respect to time interval $[0, t]$. We want to take advantage of the invariance property for non-autonomous flows in order to write (50) with respect to the time interval $[-T,-T / 2]$. To this end, we notice that, in virtue of (26), the following relations hold for all $T \in \mathbb{R}$, for all $z_{0} \in M_{z}$ and for all $t \geq 0$ :

$$
\begin{aligned}
& Z\left(\frac{T}{2}, \bar{Z}(-T), \bar{X}(\cdot-T)\right)= \\
& Z\left(-\frac{T}{2}, Z(T, \bar{Z}(-T), \bar{X}(\cdot-T)), \bar{X}(\cdot)\right)=\bar{Z}\left(-\frac{T}{2}\right) \\
& Z\left(\frac{T}{2}, z_{0}, X_{S S}^{(j)}(\cdot-T)\right)= \\
& Z\left(-\frac{T}{2}, Z\left(T, z_{0}, X_{S S}^{(j)}(\cdot-T)\right), X_{S S}^{(j)}(\cdot)\right)
\end{aligned}
$$

By using (51) and (52), the following $\delta$ ISS estimate holds for all $T \geq 0$ and for all $z_{0} \in M_{z}$ :

$$
\begin{aligned}
\left|\bar{Z}\left(-\frac{T}{2}\right)-Z\left(-\frac{T}{2}, Z\left(T, z_{0}, X_{S S}^{(j)}(\cdot-T)\right), X_{S S}^{(j)}(\cdot)\right)\right| \\
=\left|Z\left(\frac{T}{2}, \bar{Z}(-T), \bar{X}(\cdot-T)\right)-Z\left(\frac{T}{2}, z_{0}, X_{S S}^{(j)}(\cdot-T)\right)\right| \\
\quad \leq \beta\left(\left|\bar{Z}(-T)-z_{0}\right|, \frac{T}{2}\right)+ \\
\quad+\gamma\left(\mathfrak{d}_{\left[0, \frac{T}{2}\right]}\left(\bar{X}(\cdot-T), X_{S S}^{(j)}(\cdot-T)\right)\right) .
\end{aligned}
$$

Note that the following equality holds for all $T \geq 0$ :

$$
\mathfrak{d}_{\left[0, \frac{T}{2}\right]}\left(\bar{X}(\cdot-T), X_{S S}^{(j)}(\cdot-T)\right)=\mathfrak{d}_{\left[-T,-\frac{T}{2}\right]}\left(\bar{X}(\cdot), X_{S S}^{(j)}(\cdot)\right),
$$

and, due to (46), it also holds:

$$
\begin{aligned}
\lim _{T \rightarrow+\infty} & \mathfrak{d}_{\left[0, \frac{T}{2}\right]}\left(\bar{X}(\cdot-T), X_{S S}^{(j)}(\cdot-T)\right)= \\
& \lim _{T \rightarrow+\infty} \mathfrak{d}_{\left[-T,-\frac{T}{2}\right]}\left(\bar{X}(\cdot)-X_{S S}^{(j)}(\cdot)\right)=0 .
\end{aligned}
$$

In virtue of property $(\mathrm{P} 2)$, states $\bar{Z}\left(-\frac{T}{2}\right)$ and $\bar{Z}(-T)$ exist and are bounded for all $T \in \mathbb{R}$. In virtue of property (P1), states $Z\left(\frac{T}{2}, z_{0}, X_{S S}^{(j)}(\cdot-T)\right)$ and $Z\left(T, z_{0}, X_{S S}^{(j)}(\cdot-T)\right)$ exist for all $T \geq 0$ and for all $z_{0} \in M_{z}$. Therefore we can take the limit for $T \rightarrow+\infty$ in (53), which yields:

$$
\begin{aligned}
\lim _{T \rightarrow+\infty} \mid \bar{Z}\left(-\frac{T}{2}\right)- \\
Z\left(-\frac{T}{2}, Z\left(T, z_{0}, X_{S S}^{(j)}(\cdot-T)\right), X_{S S}^{(j)}(\cdot)\right) \mid=0
\end{aligned}
$$

for all $z_{0} \in M_{z}$. Pick $z_{0}$ in such a way that:

$$
\left(\begin{array}{c}
X_{S S}^{(j)}\left(-\frac{T}{2}\right) \\
Z\left(-\frac{T}{2}, Z\left(T, z_{0}, X_{S S}^{(j)}(\cdot-T)\right), X_{S S}^{(j)}(\cdot)\right)
\end{array}\right) \in \mathcal{W}_{j},
$$

for all $T \in \mathbb{R}$. This is indeed possible by selecting $z_{0}=z_{j}$ if $\mathcal{W}_{j}$ is a fixed point or by selecting $z_{0}$ as in $[1$, Proposition 4.4] if $\mathcal{W}_{j}$ is a periodic orbit. With such a choice of $z_{0}$, we can conclude from (54) that

$$
\alpha\left(\left(\begin{array}{c}
\bar{X}\left(-\frac{T}{2}\right) \\
\bar{Z}\left(-\frac{T}{2}\right)
\end{array}\right)\right) \subseteq \mathcal{W}_{j}
$$

so as to prove the Claim.

We continue with the proof of Theorem 4.6. We have shown in Claims 4.1, 4.2, and 4.3 that, for all $z_{0} \in M_{z}$, whenever $\lim _{t \rightarrow+\infty} X(t) \in \mathcal{W}_{x, i}$ it then follows:

$$
\omega\left(\left(\begin{array}{c}
X(t) \\
Z\left(t, z_{0} ; X(\cdot)\right)
\end{array}\right)\right) \subseteq \mathcal{W}_{i} .
$$

In particular, selecting $z_{0}=z_{a}$ and $X(t)=\bar{X}(t)$ yields:

$$
\omega\left(\left(\begin{array}{c}
\bar{X}(t) \\
Z\left(t, z_{a} ; \bar{X}(\cdot)\right)
\end{array}\right)\right)=\omega\left(\left(\begin{array}{c}
\bar{X}(t) \\
\bar{Z}(t))
\end{array}\right)\right) \subseteq \mathcal{W}_{i}
$$

By selecting $y_{a}=\left(x_{a}, z_{a}\right) \in \mathcal{A}\left(\mathcal{W}_{j}\right) \cap \mathcal{R}\left(\mathcal{W}_{i}\right)$, we can conclude $\mathcal{W}_{i} \prec \mathcal{W}_{j}$.

For direction $\Leftarrow$, pick $i, j=1, \ldots, N+K$ such that $\mathcal{W}_{i} \prec \mathcal{W}_{j}$ holds. Then there exists $y_{a} \in M_{x} \times M_{z}$ such that, under the flow of (7), it holds $\alpha\left(y_{a}\right)=\mathcal{W}_{j}$ and $\omega\left(y_{a}\right)=\mathcal{W}_{i}$. Given the independence of the flow of (7a) from the flow of $(7 \mathrm{~b})$, we can then select the first component of $y_{a}$, say $x_{a}$, as the point for which, under the flow of (7a), we have that $\alpha\left(x_{a}\right)=\mathcal{W}_{x, j}$ and $\omega\left(x_{a}\right)=\mathcal{W}_{x, i}$, thus satisfying $\mathcal{W}_{x, i} \prec \mathcal{W}_{x, j}$.

We are now going to rule out the existence of a 1-cycle among the atoms $\mathcal{W}_{i}$, and similar arguments will apply for the case of a $r$-cycle. By contradiction, assume that there exists a 1-cycle around $\mathcal{W}_{i}$, namely there exists an index $i$ such that $\left[\mathcal{R}\left(\mathcal{W}_{i}\right) \cap \mathcal{A}\left(\mathcal{W}_{i}\right)\right]-\mathcal{W}_{i} \neq \emptyset$. This implies the existence of $y_{a}=\left(x_{a}, z_{a}\right) \notin \mathcal{W}_{i}$ such that, under the flow of (7), $\alpha\left(y_{a}\right)=\omega\left(y_{a}\right)$.

$$
\mathcal{W}_{z, i}=\left\{Z \in M_{z} \mid Z=\bar{Z}^{(i)}(t) \text { for some } t\right\} .
$$

Two cases arise: 
- $x_{a} \notin \mathcal{W}_{x, i}$ or $X\left(\bar{t}, x_{a} ; 0\right) \notin \mathcal{W}_{x, i}$ for some $\bar{t} \in \mathbb{R}$, which implies the existence of a 1-cycle in the $x$ coordinates because, under the flow of $(7 \mathrm{a})$, we have that $\omega\left(X\left(\bar{t}, x_{a} ; 0\right)\right)=\alpha\left(X\left(\bar{t}, x_{a} ; 0\right)\right) \subseteq \mathcal{W}_{x, i}$. This immediately yields a contradiction with Assumption 3 ;

- $X\left(t, x_{a} ; 0\right) \in \mathcal{W}_{x, i}$ for all $t \in \mathbb{R}$ whereas $z_{a} \notin \mathcal{W}_{z, i}$. In virtue of property (P2) as in the proof of Claim 4.4, that there exists a unique bounded solution:

$$
Z\left(t, \cdot ; X\left(\cdot, x_{a} ; 0\right)\right)=: Z_{S S}^{(i)}(t),
$$

for all $t \in \mathbb{R}$. Since $X\left(t, x_{a} ; 0\right) \in \mathcal{W}_{x, i}$ for all $t \in \mathbb{R}$, it can be written $X\left(\cdot, x_{a} ; 0\right)=X_{S S}^{(i)}(\cdot)$ as in definition (47), for $\bar{x}^{[i]}=x_{a}$ in case of an almost periodic input signal, or some $\theta_{i}$ in case of a periodic input signal, or $x_{i}=x_{a}$ in case of a constant input signal. Correspondingly, we can define the steady-state response for all $t \in \mathbb{R}$ as:

$$
Z_{S S}^{(h)}(t):=\left\{\begin{array}{c}
Z^{[h]}\left(t, \bar{x}^{[h]}\right) \text { for } h=1, \ldots, N_{a p} \\
Z^{\left(h-N_{a p}\right)}\left(t+\frac{\theta_{h-N_{a p}}}{2 \pi} T_{h-N_{a p}}\right) \\
\quad \text { for } h=N_{a p}+1, \ldots, N_{a p}+N_{p} \\
x_{h-N_{a p}-N_{p}} \\
\text { for } h=N_{a p}+N_{p}+1, \ldots, N_{a p}+N_{p}+N_{f} .
\end{array}\right.
$$

By definitions (40), (41), and (42), $\left(X_{S S}^{(h)}(t), Z_{S S}^{(h)}(t)\right) \in$ $\mathcal{W}_{h}$ for all $t \in \mathbb{R}$, thus contradicting $\left(x_{a}, z_{a}\right) \notin \mathcal{W}_{i}$.

Step 3: ISS of the cascade system. We prove ISS of the cascaded system (6). Since $f\left(0, O_{x}\right)=0$ it is easy to check that $\delta$ ISS implies ISS just comparing an arbitrary trajectory with $Z(t) \equiv 0$ for all $t \geq 0$. Then, by virtue of Lemma Appendix A.1, we can write the asymptotic estimate (A.5) with respect to an arbitrary compact set $\tilde{\mathcal{W}} \subset M_{x} \times M_{z}$. Specifically, we can write $(\mathrm{A} .5)$ with respect to the set $\mathcal{W}_{\Theta}$. Since $\mathcal{W}_{\Theta}$ satisfies the no-cycle condition under the flow of (7), estimate (A.5) represents the practical asymptotic gain (pAG) property as introduced in [2]. By virtue of Theorem 1 in [2], we conclude ISS stability of (6) with respect to input $d$ and $\mathcal{W}_{\Phi}$.

\section{Multiperiodicity + ISS}

In this Section, we relax the assumption of incremental ISS or any contraction properties for the driven system, by only assuming ISS in the sense of [2]. Interesting complex behavior can arise from this apparently simple setting, as depicted in Figure 1. In order to simplify the analysis, we will in fact restrict our analysis to multiperiodic driving systems, namely excluding the presence of almost-periodic attractors. Then, we will derive sufficient conditions for the ISS stability of the cascade system (6).

Let $\mathcal{W}_{x}$ denote a $\mathcal{W}$-limit set of the driving system (7a). The set $\mathcal{W}_{x}$ is assumed to satisfy Assumption 3 with $N_{a p}=0$. We are looking for a $\mathcal{W}$-limit set for $(7)$ which is

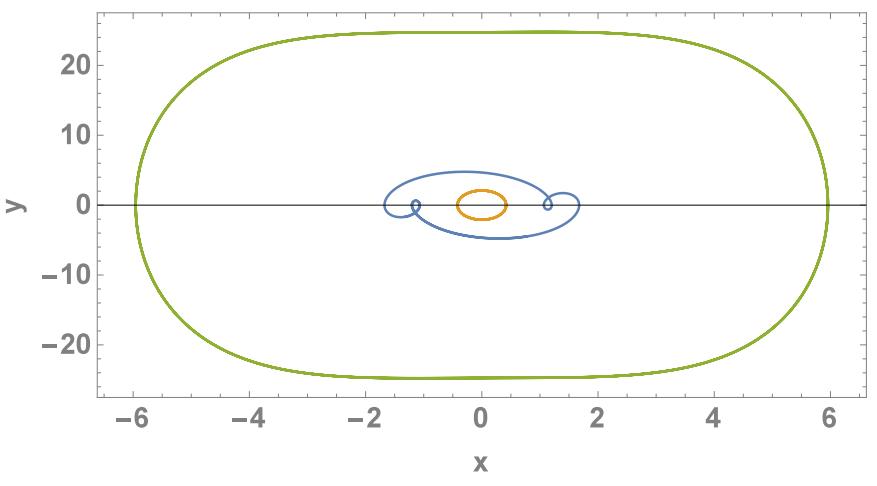

Figure 1: The Duffing oscillator $\dot{x}=y, \dot{y}=-x-x^{3}-\frac{1}{5} y+u$ can be proven to be ISS wrt the origin and input $u$ by selecting the ISS-Lyapunov function $V(x, y)=\frac{1}{2}(y+\epsilon x)^{2}+\frac{1}{2} x^{2}+\frac{1}{4} x^{4}$ with $\epsilon>0$ small enough. The plot shows the three isolated periodic orbits generated by the periodic forcing $u(t)=10 \sin (5 t)$ with different initial conditions $(x(0), y(0))=(3,-2),(0,0.1),(50,-60)$.

globally attractive and satisfies the no-cycle condition under the flow of (7). In order to provide a characterization of such set in terms of its finest possible decomposition, we again assume that any trajectory $X(t, x ; 0)$ with $x \in M_{x}$ is globally attracted to one of the $\omega$-limit sets in $\mathcal{W}_{x}$, namely either one of the equilibria $x_{i}$ s, or one of the periodic orbits $\Gamma_{x, i} \mathrm{~s}$. Thus it makes sense to:

1. study the asymptotic behavior of the driven system when its input evolves for all times on the $\omega$-limit sets of the driving system, namely $X(t, x ; 0) \in \mathcal{W}_{x, k}$ for some $k \in\left\{1, \ldots, N_{p}+N_{f}\right\}$ and for all $t \geq 0$;

2 . provide the conditions for which the asymptotic behavior observed in 1 . matches the asymptotic behavior of the driven system when its input is approaching the $\omega$-limit sets of the driving system, namely $\omega(x) \subseteq \mathcal{W}_{x, k}$

To this end, we define the asymptotically autonomous flow and the limit flow for subsystem (7b). Let $x \in M_{x}$ denote the initial condition for subsystem (7a). Assume that $X(t, x ; 0)$ is converging to one of the $\mathcal{W}_{x, k}$. In case $k \in\left\{N_{p}+1, \ldots, N_{p}+N_{f}\right\}$, the set $\mathcal{W}_{x, k}$ is the equilibrium $\left\{x_{k-N_{p}}\right\}$, and we have $X(t, x ; 0) \rightarrow x_{k-N_{p}}$ as $t \rightarrow+\infty$. Hence, we will consider for each $k \in\left\{N_{p}+1, \ldots, N_{p}+N_{f}\right\}$ the (time-varying) asymptotically autonomous system:

$$
\dot{z}(t)=F_{k}(z(t), t):=f(z(t), X(t, x ; 0)),
$$

and its corresponding (time-invariant) limit system:

$$
\dot{z}(t)=\bar{F}_{k}(z(t)):=f\left(z(t), x_{k-N_{p}}\right),
$$

In case $k \in\left\{1, \ldots, N_{p}\right\}$, the set $\mathcal{W}_{x, k}$ is the periodic orbit $\Gamma_{x, k}$. Note that

$$
\mathfrak{d}\left(X(t, x ; 0), X^{(k)}\left(t+\frac{\theta}{2 \pi} T_{k}\right)\right) \text { as } t \rightarrow+\infty
$$

for some $\theta \in[0,2 \pi)$. In this case we will consider for each $k \in\left\{1, \ldots, N_{p}\right\}$ the (time-varying) asymptotically 
autonomous system in the extended state-space $\tilde{z}=(\tau, z) \in$ $\mathbb{S} \times M_{z}$ :

$$
\dot{\tilde{z}}(t)=\left(\begin{array}{c}
\dot{\tau}(t) \\
\dot{z}(t)
\end{array}\right)=F_{k}(z(t), t)=\left(\begin{array}{c}
\frac{2 \pi}{T_{k}} \\
f(z(t), X(t, x ; 0))
\end{array}\right),
$$

and its corresponding (time-invariant) limit system:

$$
\dot{\tilde{z}}(t)=\left(\begin{array}{c}
\dot{\tau}(t) \\
\dot{z}(t)
\end{array}\right)=\bar{F}_{k}(\tilde{z}(t), t)=\left(\begin{array}{c}
\frac{2 \pi}{T_{k}} \\
f\left(z(t), X^{(k)}(\tau(t))\right)
\end{array}\right) .
$$

It can be easily seen that the initial condition on $\tau$ can be set equal to the phase $\theta$ of the periodic orbit $X^{(k)}\left(t+\frac{\theta}{2 \pi} T_{k}\right)$ asymptotically approached by $X(t, x ; 0)$. In other words, we can study the asymptotic behavior of the periodically forced subsystem (7b) with different phases by simply considering the extended state-space $\mathbb{S} \times M_{z}$ and changing the initial condition $\tau$.

The following key assumption is made on each limit system. For $k=N_{p}+1, \ldots, N_{p}+N_{f}$, let $\mathcal{W}_{z}^{(k)}$ denote a $\mathcal{W}$-limit set of limit equation (56) with the finest possible decomposition. For $k=1, \ldots, N_{p}$, let $\mathcal{W}_{\tilde{z}}^{(k)}$ denote a $\mathcal{W}$ limit set of limit equation (58) with the finest possible decomposition.

Assumption 5. (Limit systems without cycles) For all $k=1, \ldots, N_{p}$, the limit equation (58), thus each set $\mathcal{W}_{\tilde{z}}^{(k)}$ satisfies the no-cycle condition. For all $k=N_{p}+1, \ldots, N_{p}+$ $N_{f}$, the limit equation (56), thus each set $\mathcal{W}_{z}^{(k)}$ satisfies the no-cycle condition. For all $k=1, \ldots, N_{p}+N_{f}$, we denote with $N_{k}$ the number of atoms in the decomposition of $\mathcal{W}_{z}^{(k)}$.

Our aim is to find a $\mathcal{W}$-limit set for (7) which is globally attractive and satisfies the no-cycle condition under the flow of (7). To this end, we note that each solution of (58) which evolves on an atom $\mathcal{W}_{\tilde{z}, h}^{(k)} \subseteq \mathcal{W}_{\tilde{z}}^{(k)}$ for all $t \geq 0$ and some $h \in\left\{1, \ldots, N_{p}\right\}$ can be defined by selecting any initial condition $\left(\tau_{k, h}, z_{k, h}\right) \in \mathcal{W}_{\tilde{z}, h}^{(k)}$ and by denoting, for all $t \geq 0$,

$$
\tilde{Z}_{h}^{(k)}\left(t,\left(\tau_{k, h}, z_{k, h}\right)\right):=\left(\begin{array}{c}
{\left[\tau_{k, h}+\frac{2 \pi}{T_{k}} t\right] \bmod 2 \pi} \\
Z_{h}^{(k)}\left(t,\left(\tau_{k, h}, z_{k, h}\right)\right)
\end{array}\right),
$$

where $Z_{h}^{(k)}\left(t,\left(\tau_{k, h}, z_{k, h}\right)\right)$ simply denotes the projection of $\tilde{Z}_{h}^{(k)}\left(t,\left(\tau_{k, h}, z_{k, h}\right)\right)$ onto the $M_{z}$ manifold. It is then immediate to define the following invariant sets for the cascade dynamics $(7)$ :

$$
\mathcal{W}^{(k, h)}:=\left\{\left(\begin{array}{c}
X^{(k)}\left(t+\frac{\tau_{k, h}}{2 \pi} T_{k}\right) \\
Z_{h}^{(k)}\left(t,\left(\tau_{k, h}, z_{k, h}\right)\right)
\end{array}\right) \in M_{x} \times M_{z}, t \geq 0\right\},
$$

with $k=1, \ldots, N_{p}$ and $h=1, \ldots, N_{k}$. Observe that the definition of set $\mathcal{W}^{(k, h)}$ does not depend upon the choice of initial condition $\left(\tau_{k, h}, z_{k, h}\right)$. We can also define the set $\mathcal{W}^{(k, h)}$ for $k=N_{p}+1, \ldots, N_{p}+N_{f}$ and $h=1, \ldots, N_{k}$, as follows:

$$
\mathcal{W}^{(k, h)}:=\left\{x_{k}\right\} \times \mathcal{W}_{z, h}^{(k)}
$$

Therefore, a natural question that arises from this setting is whether the sought $\mathcal{W}$-limit set for the cascade system (7) can be selected as the set:

$$
\mathcal{W}_{\Theta}:=\bigcup_{k=1}^{N_{p}+N_{f}} \bigcup_{h=1}^{N_{k}} \mathcal{W}^{(k, h)}
$$

In order to answer the question, we recall the following result due to Mischaikow, Smith and Thieme in [8, Theorem 1.8]: whenever the asymptotically autonomous semiflow (55)-(57) verifies boundedness of trajectories, its $\omega$-limit sets are subsets of the compact, connected subsets of $M_{z}$ which are invariant and chain recurrent for (56)-(58) respectively.

Theorem 5.1. Let Assumptions 3 and 5 hold with $N_{a p}=$ 0. If:

- the driving system (6a) is ISS wrt input $d$ and the invariant set $\mathcal{W}_{x}$;

- the driven system (6b) is ISS wrt input $|X(\cdot)|$ and some invariant set $\mathcal{W}_{z}$;

then the cascade system (6) is ISS wrt input $d$ and the invariant set $\mathcal{W}_{\Theta}$. Moreover, a filtration ordering of $\mathcal{W}_{\Theta}$ under the flow of (6) is inherited by the filtration orderings of $\mathcal{W}_{x}$ as follows:

$$
\mathcal{W}_{\tilde{z}, i}^{(k)} \prec \mathcal{W}_{\tilde{z}, j}^{(k)} \Leftrightarrow \mathcal{W}^{(k, i)} \prec \mathcal{W}^{(k, j)},
$$

for all $k \in\left\{1, \ldots, N_{p}+N_{f}\right\}$ and all $i, j \in\left\{1, \ldots, N_{k}\right\}$;

Proof. The proof follows along the lines of [5] and of Theorem 3.1. Main steps of the proof:

- Lemma Appendix A.1 yields the practical asymptotic gain (pAG) property with respect to an arbitrary compact set $\tilde{\mathcal{W}} \in M_{x} \times M_{z}$, hence boundedness of trajectories for the unperturbed cascade system (7);

- due to boundedness of trajectories and Assumption 5 , it is possible to apply the results of Theorem 1.8 in [8] to conclude that the set $\mathcal{W}_{\Theta}$ as defined in (60) is globally attractive for the flow of the cascade (7);

- the set $\mathcal{W}_{\Theta}$ satisfies the no-cycle condition under the flow of cascade (7), and a proof of this claim together with the proof of claim (61) follows along the lines of Theorem 3.1;

- given the pAG property and the fact that $\mathcal{W}_{\Theta}$ qualifies itself as a $\mathcal{W}$-limit set for (7), Claim 4 in [2] yields the AG property of the cascade (6) wrt to input $d$ and the set $\mathcal{W}_{\Theta}$.

Corollary 5.1. Let Assumptions 3 and 5 hold with $N_{a p}=$ 0. If: 
- the driving system ( 7 a) has an invariant set $\mathcal{W}_{x}$ which is globally attractive;

- the driven system (7b) is ISS wrt input $|X(\cdot)|$ and some invariant set $\mathcal{W}_{z}$;

then the invariant set $\mathcal{W}_{\Theta}$ is globally attractive for the cascade system (7) and satisfies the no-cycle condition for (7).

\section{Examples}

\subsection{Multistability of driving system}

As an example of an ISS system driven by a multistable system, consider the following cascade system:

$$
\begin{aligned}
& \dot{x}=-x^{3}+x+d \\
& \dot{z}=-\sin (z)+x,
\end{aligned}
$$

with states $x \in \mathbb{R}$ and $z \in \mathbb{S}$ and disturbance $d \in \mathbb{R}$. For subsystem (62a), the decomposable $\mathcal{W}$-limit set takes the form: $\mathcal{W}_{x}=\mathcal{W}_{x, 1} \cup \mathcal{W}_{x, 2} \cup \mathcal{W}_{x, 3}=\{-1,1,0\}$. The Lyapunov function $V_{x}(x)=(x-1)^{2}(x+1)^{2}$ satisfies:

$$
\begin{aligned}
|x|_{\mathcal{W}_{x}}^{4} & \leq V_{x}(x) \\
D V_{x}(x)\left(-x^{3}+x+d\right) & \leq-|x|_{\mathcal{W}_{x}}^{6}+4 d^{2},
\end{aligned}
$$

and hence shows that $(62 \mathrm{a})$ is ISS wrt $\mathcal{W}_{x}$ and $|d|$. For subsystem $(62 \mathrm{~b})$ with input $x$, the decomposable $\mathcal{W}$-limit set takes the form: $\mathcal{W}_{z}^{(3)}=\mathcal{W}_{z, 1}^{(3)} \cup \mathcal{W}_{z, 2}^{(3)}=\{0, \pi\}$. The Lyapunov function $V_{z}(z)=1-\cos (z)$ satisfies:

$$
\begin{aligned}
& 0.1|z|_{\mathcal{W}_{z}^{(3)}}^{2} \leq V_{z}(z) \\
& D V_{z}(z)(-\sin (z)+x)=-\sin (z)^{2}+x \sin (z) \\
& -\frac{1}{2} \sin (z)^{2}+\frac{1}{2} x^{2} \leq-0.15|z|_{\mathcal{W}_{z}^{(3)}}^{2}+\frac{1}{2} x^{2},
\end{aligned}
$$

and hence shows that $(62 \mathrm{a})$ is ISS wrt $\mathcal{W}_{z}^{(3)}$ and $|x|$. In virtue of Theorem 3.1, we consider the $\alpha$ - and $\omega$-limit sets of the limit equations for each equilibrium $x_{i}, i=$ $1, \ldots, 3$ : Case $i=1$ : the solutions of the limiting system $\dot{z}=-\sin (z)-1$ all converge to the equilibrium $\{\pi / 2\}$. This is not asymptotically stable. In fact it exhibits a homoclinic cycle. Therefore, in order to satisfy Assumption 2 we need to consider an enlarged invariant set, namely $\mathcal{W}_{z}^{(1)}=\mathbb{S}$. Case $i=2$ : same arguments hold true for equation $\dot{z}=-\sin (z)+1$, hence $\mathcal{W}_{z}^{(2)}=\mathbb{S}$. Case $i=3$ : for equation $\dot{z}=-\sin (z)$ we select $\mathcal{W}_{z}^{(3)}=\{0, \pi\}$. In virtue of Theorem 3.1, we can conclude that the set

$$
\mathcal{W}_{\Theta}=(\{-1\} \times \mathbb{S}) \cup(\{1\} \times \mathbb{S}) \cup\{(0,0)\} \cup\{(0, \pi)\}
$$

qualifies itself as a $\mathcal{W}$-limit set for (62) and, moreover, system (62) is ISS wrt $\mathcal{W}_{\Theta}$ and $d$.

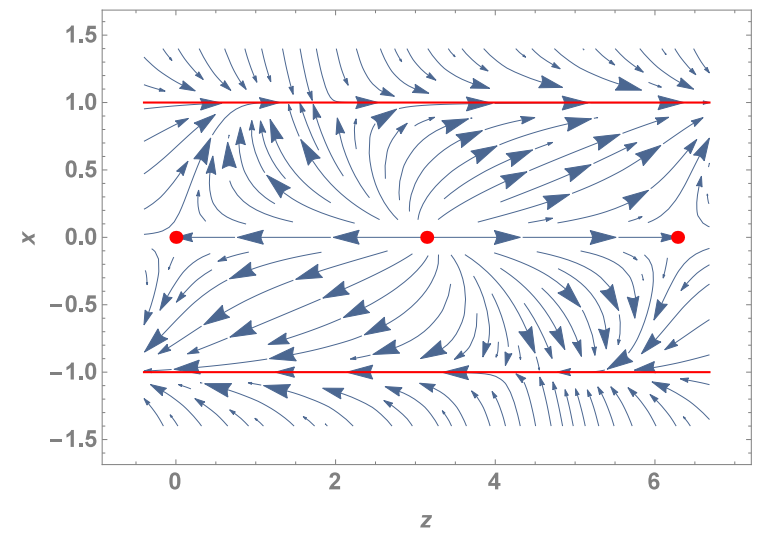

Figure 2: Phase plot of dynamics (62). The set $\mathcal{W}_{\Theta}$ in $(63)$ is in red.

\subsection{Multiperiodicity of driving system - Robotics}

As an example of an incrementally ISS system driven by an oscillating source, we study here the case of a robotic manipulator tracking a trajectory provided by the so-called Central Pattern Generators (CPGs). CPGs represent a powerful method for the robust generation of rhythmic patterns and for this reason they are widely used in locomotion control of robots [6]. Consider a 3 degrees-offreedom mechanical manipulator whose motion is described by the equations:

$$
M(q) \ddot{q}+C(q, \dot{q}) \dot{q}+G(q)=\tau,
$$

with $q, \dot{q}, \ddot{q} \in \mathbb{R}^{3}$ the joint angles, velocities and accelerations, $M(q)$ the inertia matrix, $C(q, \dot{q})$ the Coriolis matrix, $G(q)$ the potential vector field, $\tau$ the vector of all available control torques, and $d$ disturbances occurring in the joint dynamics. The standard computed torque control algorithm is implemented on (64):

$$
\begin{aligned}
& \tau=M(q) \nu+C(q, \dot{q}) \dot{q}+G(q) \\
& \nu=q_{r(2)}+K_{d}\left(q_{r(1)}-\dot{q}\right)+K_{p}\left(q_{r}-q\right),
\end{aligned}
$$

where $K_{d}=\operatorname{diag}\left[k_{d 1}, k_{d 2}, k_{d 3}\right]$ and $K_{p}=\operatorname{diag}\left[k_{p 1}, k_{p 2}, k_{p 3}\right]$ are tunable controller gains. In (65), variables

$$
q_{r}=\left(\begin{array}{l}
q_{r 1} \\
q_{r 2} \\
q_{r 3}
\end{array}\right), q_{r(1)}=\left(\begin{array}{l}
q_{r 1(1)} \\
q_{r 2(1)} \\
q_{r 3(1)}
\end{array}\right), q_{r(2)}=\left(\begin{array}{c}
q_{r 1(2)} \\
q_{r 2(2)} \\
q_{r 3(1)}
\end{array}\right)
$$

respectively denote the reference trajectory and its filtered first and second time derivative, as specified in the following:

$$
\frac{q_{r i(1)}(s)}{q_{r i}(s)}=\frac{p s}{p+s}, \quad \frac{q_{r i(2)}(s)}{q_{r i}(s)}=\frac{p s^{2}}{(p+s)^{2}},
$$

with $i=1,2,3$ and $p>0$ the tunable pole of the filter. By combining equations (64), (65), and a minimal statespace representation of (67), we obtain the following linear system for each joint $i=1,2,3$ :

$$
\dot{z}=A z+B q_{r}
$$


with

$$
A=\left[\begin{array}{cccc}
-p & 0 & 0 & 0 \\
-p^{2} & -p & 0 & 0 \\
0 & 0 & 0 & 1 \\
k_{d i}+p & 1 & -k_{p i} & -k_{d i}
\end{array}\right], B=\left[\begin{array}{c}
-p^{2} \\
-p^{3} \\
0 \\
p^{2}+k_{p i}+k_{d i} p
\end{array}\right]
$$

The reference trajectory $q_{r}$ for the torque-controlled manipulator is generated by the CPGs, which are implemented as a set of coupled nonlinear oscillators (typically $\mathrm{Ku}-$ ramoto model), as follows:

$$
\begin{aligned}
\dot{\theta_{1}} & =\omega+d_{1}+K \sin \left(\theta_{2}-\theta_{1}\right)+K \sin \left(\theta_{3}-\theta_{1}+\pi\right) \\
\dot{\theta_{2}} & =\omega+d_{2}+K \sin \left(\theta_{1}-\theta_{2}\right)+K \sin \left(\theta_{3}-\theta_{2}+\pi\right) \\
\dot{\theta_{3}} & =\omega+d_{3}+K \sin \left(\theta_{1}-\theta_{3}-\pi\right)+K \sin \left(\theta_{2}-\theta_{3}-\pi\right) \\
q_{r i} & =\bar{q}_{i}+\hat{q}_{i} \cos \left(\theta_{i}\right),
\end{aligned}
$$

where $\theta_{i} \in \mathbb{S}$ denotes the phase of oscillator $i=1,2,3$, $\omega>0$ is the common frequency for all oscillators, $d_{i} \in \mathbb{R}$ represents a generic disturbance, $K>0$ is the coupling strength, and $\bar{q}_{i}, \hat{q}_{i} \in \mathbb{R}$ respectively denote bias and amplitude of the oscillation driving joint $i=1,2,3$. Model (69) has been studied in [2] where the authors have shown that, by rewriting the dynamics wrt the synchronization errors $e_{1}:=\theta_{2}-\theta_{1}, e_{2}:=\theta_{3}-\theta_{1}+\pi$, the $\mathcal{W}$-limit set for the transformed dynamics takes the form:

$$
\mathcal{W}_{e}=\left\{(0,0),(0, \pi),(\pi, 0),(\pi, \pi),\left(\frac{2 \pi}{3}, \frac{4 \pi}{3}\right),\left(\frac{4 \pi}{3}, \frac{2 \pi}{3}\right)\right\}
$$

and, moreover, satisfies the no cycle condition (2.4), as shown in Figure 3. This in turn implies that the $\mathcal{W}$-limit set $\mathcal{W}_{x}$ of the original system (69) consists of periodic orbits in a one-to-one correspondence with the elements of (70) and satisfies the no cycle condition.

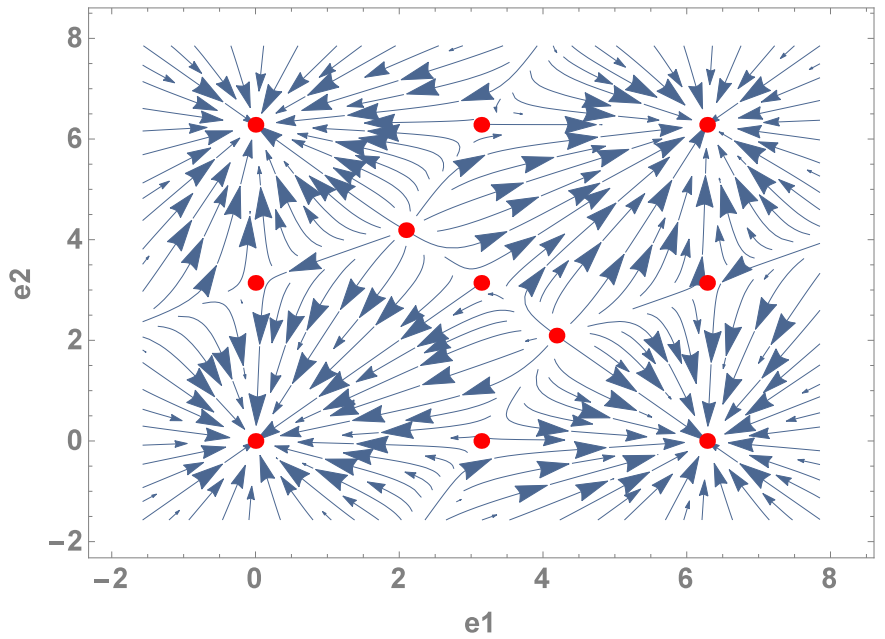

Figure 3: Phase plot of dynamics (69) in the coordinates $e_{1}, e_{2}$. The set $\mathcal{W}_{e}$ as in (70) is depicted in red.

The authors in [2] have proved that system (69) is ISS wrt to disturbances $d_{i}$ and the set $\mathcal{W}_{x}$. It can be easily proved that system $\dot{z}=A z+B\left(\bar{q}_{i}+\hat{q}_{i} \cos \left(\theta_{i}\right)\right)$ is incrementally ISS wrt to input $\theta_{i}$ by noticing that it is a linear system and $\left|\cos \left(\theta_{i}\right)\right| \leq 1$ is bounded. Therefore the results of Theorem 4.6 can be applied to infer the ISS property for the cascade $(68), 69)$ wrt disturbances $d_{i}$.

\subsection{Multiperiodic subsystem driving ISS subsystem}

The following example is a straightforward application of Corollary 5.1. Consider the following set of differential equations:

$$
\begin{aligned}
& \left(\begin{array}{c}
\dot{x}_{1} \\
\dot{x}_{2}
\end{array}\right)=\left(\begin{array}{c}
-x_{2} \\
x_{1}
\end{array}\right)+ \\
& \quad+\left(\begin{array}{c}
x_{1} \\
x_{2}
\end{array}\right)\left(1-x_{1}^{2}-x_{2}^{2}\right)\left(\frac{9}{4}-x_{1}^{2}-x_{2}^{2}\right)\left(4-x_{1}^{2}-x_{2}^{2}\right) \\
& \dot{z}=-z^{3}+z\left(1+\sin \left(x_{1}\right)\right) .
\end{aligned}
$$

The driving subsystem $(71)$ has a $\mathcal{W}$-limit set which can be decomposed as follows:

$$
\mathcal{W}_{x}=\mathcal{W}_{x, 1} \cup \mathcal{W}_{x, 2} \cup \mathcal{W}_{x, 3} \cup \mathcal{W}_{x, 4}=\Gamma_{x, 1} \cup \Gamma_{x, 2} \cup \Gamma_{x, 3} \cup\left\{x_{4}\right\}
$$

$$
\text { with } \begin{aligned}
\Gamma_{x, 1} & =\left\{\left(x_{1}, x_{2}\right) \in \mathbb{R}^{2} \mid x_{1}^{2}+x_{2}^{2}=1\right\} \\
\Gamma_{x, 2} & =\left\{\left(x_{1}, x_{2}\right) \in \mathbb{R}^{2} \mid x_{1}^{2}+x_{2}^{2}=\frac{9}{4}\right\} \\
\Gamma_{x, 3} & =\left\{\left(x_{1}, x_{2}\right) \in \mathbb{R}^{2} \mid x_{1}^{2}+x_{2}^{2}=2\right\} .
\end{aligned}
$$

In particular, the Poincaré map of system (71) proves that $\Gamma_{x, 1}$ and $\Gamma_{x, 3}$ are locally asymptotically stable periodic orbits, whereas $\Gamma_{x, 2}$ is a repelling one, while $x_{4}=(0,0)$ is repelling fixed point. Thus, the set $\mathcal{W}_{x}$ qualifies as a $\mathcal{W}$ limit set for subsystem (71). Moreover, a trajectory lying on the limit cycles $\Gamma_{x, i}$ with $i=1, \ldots, 3$ takes the form:

$$
\left(\begin{array}{l}
x_{1}(t) \\
x_{2}(t)
\end{array}\right)=\left(\begin{array}{l}
A_{i} \sin (t+\theta) \\
A_{i} \cos (t+\theta)
\end{array}\right)
$$

where $A_{1}=1, A_{1}=2$, and $A_{3}=3 / 2$. Subsystem $\dot{z}=$ $-z^{3}+z u$ is proven to be ISS by the ISS-Lyapunov function $V_{z}(z)=z^{2}$. Note that subsystem $\dot{z}=-z^{3}+z u$ satisfies the property that the sets $\mathbb{R}_{>0}, \mathbb{R}_{<0}$, and $\{0\}$ are invariant for all times regardless of the input function $u(t)$.

We are now going to check that Assumption 5 is indeed satisfied for subsystem (72). We then study the limit systems (58) in the extended state-space $\mathbb{S} \times M_{z}$ for each periodic orbit (74). In particular, we study the limit system corresponding to $\Gamma_{x, 1}$, namely:

$$
\begin{aligned}
& \dot{\tau}=1 \\
& \dot{z}=-z^{3}+z(1+\sin (\tau)),
\end{aligned}
$$

and a similar analysis will hold true for $\Gamma_{x, 2}$ and $\Gamma_{x, 3}$. The phase plot of dynamics (75), as depicted in Figure 4, shows that the limit system (75) has 3 invariant sets which are not cyclically chained, as proved in the following. The Poincaré map

$$
(x(0), \theta(0) \equiv 0) \mapsto(x(2 \pi), \theta(0)+2 \pi \equiv 0),
$$




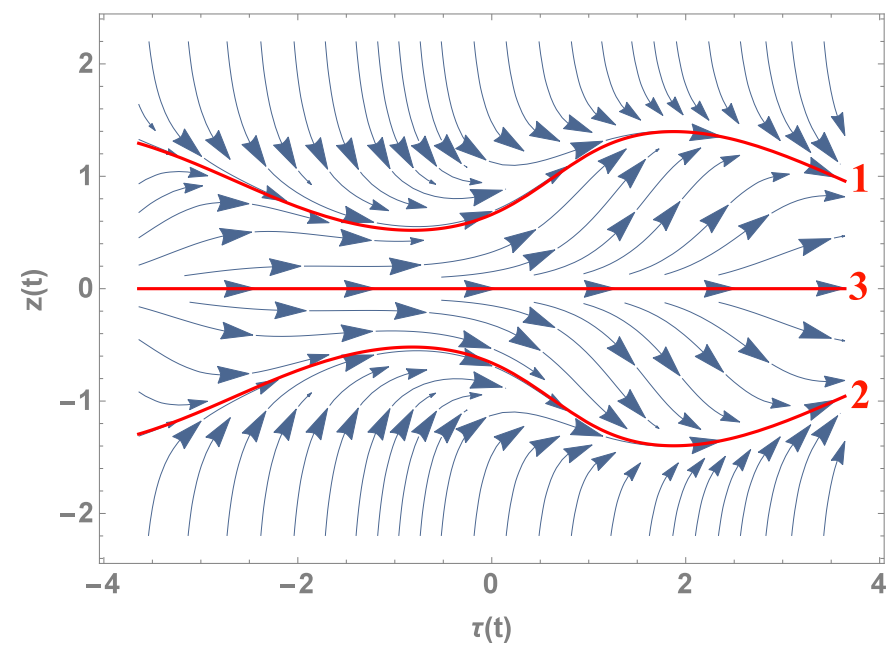

Figure 4: Phase plot of dynamics $(75)$ and corresponding $\mathcal{W}$-limit set (red).

can be computed analytically by means of software tools. Indeed, a numerical approximation for such a map when $x(0)>0$ is:

$$
x(2 \pi) \cong \frac{196.9963}{\sqrt{89026.7678+\frac{0.1353}{x_{0}^{2}}}} .
$$

Map (76) has an equilibrium at $\bar{x} \cong 0.6602$ which is asymptotically stable with basin of attraction $\mathbb{R}_{>0}$. For the case $x(0)<0$, the numerical approximation of the Poincarè map shows that the equilibrium at $\bar{x}_{1} \cong-0.6602$ is asymptotically stable with basin of attraction $\mathbb{R}_{<0}$. Hence, we can conclude that the 3 periodic orbits of $(75)$ are indeed not cyclically chained. For the periodic orbit of the driving system

$$
X^{(1)}(t)=\left(\begin{array}{c}
\sin (t) \\
\cos (t)
\end{array}\right)
$$

these periodic orbits are:

$$
\begin{aligned}
& \tilde{Z}_{1}^{(1)}(t)=\left(\begin{array}{c}
t \\
Z_{1}^{(1)}\left(t,\left(\bar{x}_{1}, 0\right)\right)
\end{array}\right) \\
& \tilde{Z}_{2}^{(1)}(t)=\left(\begin{array}{c}
t \\
Z_{2}^{(1)}\left(t,\left(-\bar{x}_{1}, 0\right)\right)
\end{array}\right)=\left(\begin{array}{c}
t \\
-Z_{1}^{(1)}\left(t,\left(\bar{x}_{1}, 0\right)\right)
\end{array}\right), \\
& \tilde{Z}_{3}^{(1)}(t)=\left(\begin{array}{c}
t \\
Z_{3}^{(1)}(t,(0,0))
\end{array}\right)=\left(\begin{array}{l}
t \\
0
\end{array}\right) .
\end{aligned}
$$

for some periodic orbit $\left.Z_{1}^{(1)}\left(t, \bar{x}_{1}, 0\right)\right)$ A similar analysis shows that, for each periodic orbit (74) of the driving subsystem (71) there exist 3 periodic orbits of the limit system (58) which are not cyclically chained. In particular, for $X^{(2)}(t)=(2 \sin (t), 2 \cos (t))$ we have

$$
\begin{aligned}
& Z_{1}^{(2)}\left(t,\left(\bar{x}_{2}, 0\right)\right), Z_{2}^{(2)}\left(t,\left(-\bar{x}_{2}, 0\right)\right) \equiv-Z_{1}^{(2)}\left(t,\left(\bar{x}_{2}, 0\right)\right), \\
& Z_{3}^{(2)}(t, 0)=(t, 0)
\end{aligned}
$$

and for $X^{(3)}(t)=\left(\frac{3}{2} \sin (t), \frac{3}{2} \cos (t)\right)$ we have

$$
\begin{aligned}
& Z_{1}^{(3)}\left(t,\left(\bar{x}_{3}, 0\right)\right), Z_{2}^{(3)}\left(t,\left(-\bar{x}_{3}, 0\right)\right) \equiv-Z_{1}^{(3)}\left(t,\left(\bar{x}_{3}, 0\right)\right), \\
& \left.Z_{3}^{(3)}(t, 0)=t, 0\right) .
\end{aligned}
$$

Moreover, the origin of the unperturbed driven system (72) is globally attractive, thus we can define the set $\mathcal{W}^{(4,1)}=$ $(0,0,0)$ which is invariant under the flow of the cascade (71)-(72). The remaining invariant sets are defined as: $\mathcal{W}^{(k, h)}=\left(\begin{array}{c}X^{(k)}(t) \\ Z_{h}^{k}\end{array}\right)$ for all $k=1, \ldots, 3$ and all $h=1, \ldots, 3$.

Therefore, the $\mathcal{W}$-limit set for the cascade dynamics (71)(72) takes the form:

$$
\mathcal{W}_{\Theta}=\mathcal{W}^{(4,1)} \cup \bigcup_{k=1}^{3} \bigcup_{h=1}^{3} \mathcal{W}^{(k, h)}
$$

\section{Conclusions}

Based on a novel ISS framework for systems with multiple not-necessarily-connected invariant sets, we have studied the cascade interconnections of ISS systems exhibiting typical nonlinear behaviors (multistability, periodic, and almost-periodic and non-periodic orbits). We have shown that the ISS property is conserved in cascaded systems whenever specific sufficient conditions are satisfied. In particular, we have provided a characterization of the $\mathcal{W}$-limit set (invariant set containing all $\alpha$ - and $\omega$-limit sets) of such cascade system in terms of its finest possible decomposition. We remark that among the sufficient conditions yielding ISS for the cascade, the absence of cycles in each component of the cascade turns out to be a very important one. Indeed, without such assumption, no appropriate definition of the decomposition of the $\mathcal{W}$-limit set of the cascade can be made and, furthermore, ISS of each subsystem only guarantees boundedness of trajectories of the cascade.

Hopefully, this paper will serve as a starting point for the analysis of the stability and sensitivity to disturbances for many systems of interests, such as those arising in the field of systems biology.

\section{Appendix A. Technical lemmas}

The following two lemmas are obtained by adapting the arguments in the proof of Theorem 2 in [13].

Lemma Appendix A.1. If:

- for the driving system (6a) there exists a positivedefinite and proper function $V_{x}$, functions $\alpha_{x, 1}, \alpha_{x, 2}$, $\alpha_{x}, \gamma_{x} \in \mathcal{K}_{\infty}$, and positive constants $c_{x}, q_{x}$, which satisfy the inequalities:

$$
\begin{aligned}
& \alpha_{x, 1}\left(|x|_{\mathcal{W}_{x}}\right) \leq V_{x}(x) \leq \alpha_{x, 2}\left(|x|_{\mathcal{W}_{x}}+c_{x}\right) \\
& \frac{\partial}{\partial x} V_{x}(x) g(x, d) \leq-\alpha_{x}\left(|x|_{\mathcal{W}_{x}}\right)+\gamma_{x}(|d|)+q_{x},
\end{aligned}
$$

with respect to an arbitrary compact set $\mathcal{W}_{x} \subset M_{x}$; 
- for the driven system (6b) there there exist a positivedefinite and proper function $V_{z}$, functions $\alpha_{z, 1}, \alpha_{z, 2}$, $\alpha_{z}, \gamma_{z} \in \mathcal{K}_{\infty}$, and a positive constant $c_{z}, q_{z}$, such that:

$$
\begin{aligned}
& \alpha_{z, 1}\left(|z| \mathcal{W}_{z}\right) \leq V_{z}(z) \leq \alpha_{z, 2}\left(|z|_{\mathcal{W}_{z}}+c_{z}\right) \\
& \frac{\partial}{\partial z} \dot{V}_{z}(z) f(z, x) \leq-\alpha_{z}\left(|z|_{\mathcal{W}_{z}}\right)+\gamma_{z}(|x|)+q_{z},
\end{aligned}
$$

with respect to an arbitrary compact set $\mathcal{W}_{z} \subset M_{z}$;

then the cascade system (6) enjoys the practical AG property with respect to an arbitrary compact set $\tilde{\mathcal{W}} \subset M_{x} \times$ $M_{z}$, namely it holds that

$$
\limsup _{t \rightarrow+\infty}|Y(t, y ; d)|_{\tilde{\mathcal{W}}} \leq \sigma^{\prime}(\|d\|)+q^{\prime}
$$

for some $\mathcal{K}_{\infty}$ function $\sigma^{\prime}$ and positive constant $q^{\prime}$.

Proof. Due to compactness of the set $\mathcal{W}_{x}$, the following bounds hold:

$$
|x| \leq \nu_{3}\left(|x|_{\mathcal{W}_{x}}\right)+c_{3}, \quad|x|_{\mathcal{W}_{x}} \leq \nu_{4}(|x|)+c_{4},
$$

for some $\mathcal{K}_{\infty}$ function $\nu_{3}, \nu_{4}$ and some positive constant $c_{3}, c_{4}$. Let $\tilde{\beta}(s)$ be any class $\mathcal{K}_{\infty}$ and smooth function such that:

$$
\tilde{\beta}(s):= \begin{cases}\alpha_{x}(s) & \text { in a neighborhood of } 0 \\ \gamma_{z}\left(2 \nu_{3}(s)\right) & \text { in a neighborhood of }+\infty .\end{cases}
$$

It then follows that $\tilde{\beta}(s)=\mathcal{O}\left(\alpha_{x}(s)\right)$ as $s \rightarrow 0^{+}$, and $\gamma_{z}(s)=\mathcal{O}\left(\frac{1}{2} \tilde{\beta}(s)\right)$ as $s \rightarrow+\infty$. By virtue of Lemma Appendix A.2, there exists a positive definite and proper function $\tilde{V}_{x}(x)$, a $\mathcal{K}_{\infty}$ function $\tilde{\gamma}_{x}$, and a positive constant $\tilde{q}_{x}$ such that inequality (A.2) can be rewritten as:

$$
\dot{\tilde{V}}_{x}(x) \leq-\tilde{\beta}_{x}\left(|x|_{\mathcal{W}_{x}}\right)+\tilde{\gamma}_{x}(|d|)+\tilde{q}_{x}
$$

Moreover, by noting that

$$
\gamma_{z}(|x|) \leq \gamma_{z}\left[\nu_{3}\left(|x|_{\mathcal{W}_{x}}\right)+c_{3}\right] \leq \gamma_{z}\left(2 \nu_{3}\left(|x|_{\mathcal{W}_{x}}\right)\right)+\gamma_{z}\left(2 c_{3}\right),
$$

inequality (A.4) can be rewritten as:

$$
\frac{\partial V_{z}(z)}{\partial z} f(z, x) \leq-\alpha_{z}\left(|z|_{\mathcal{W}_{z}}\right)+\gamma_{z}\left(2 \nu_{3}|x|_{\mathcal{W}_{x}}\right)+\gamma_{z}\left(2 c_{3}\right)+q_{z} .
$$

In virtue of Lemma Appendix A.3, there exists a positive definite and proper function $\tilde{V}_{z}(z)$, a $\mathcal{K}_{\infty}$ function $\tilde{\alpha}_{z}$, and a positive constants $\tilde{q}_{z}$ such that inequality (A.7) can be rewritten as:

$$
\dot{\tilde{V}}_{z}(z) \leq-\tilde{\alpha}_{z}\left(|z|_{\mathcal{W}_{z}}\right)+\frac{1}{2} \tilde{\beta}\left(|x|_{\mathcal{W}_{x}}\right)+\tilde{q}_{x} .
$$

Then, adding inequalities (A.6) and (A.8) yields the following estimate:

$\dot{\tilde{V}}_{x}(x, d)+\dot{\tilde{V}}_{z}(z, x) \leq-\frac{1}{2} \tilde{\beta}_{x}\left(|x|_{\mathcal{W}_{x}}\right)-\alpha_{z}\left(|x|_{\mathcal{W}_{z}}\right)+\tilde{q}_{x}+\tilde{q}_{z}$.
Consider now the extended state $y=(x, z)$ and the set $\mathcal{W}_{y}:=\mathcal{W}_{x} \times \mathcal{W}_{z}$. Note that the function $V(y):=V_{x}(x)+$ $V_{z}(z)$ satisfies the estimates:

$$
\begin{gathered}
\alpha_{1}\left(|y|_{\mathcal{W}_{y}}\right) \leq V(y) \leq \alpha_{2}\left(|x|_{\mathcal{W}_{y}}+c_{y}\right) \\
\dot{V}(y) \leq-\alpha\left(|y|_{\mathcal{W}_{y}}\right)+\tilde{\gamma}_{x}(|d|)+q_{y},
\end{gathered}
$$

for some $\mathcal{K}_{\infty}$ function $\alpha, \alpha_{1}, \alpha_{2}$, and positive constants $c_{y}, q_{y}$. Inequalities (A.9) and (A.10) in turn implies an estimate as follows:

$$
|Y(t, y ; d)|_{\mathcal{W}_{y}} \leq \kappa\left(|y|_{\mathcal{W}_{y}}, t\right)+\sigma(\|d\|)+q \forall t \geq 0
$$

where $\kappa \in \mathcal{K} \mathcal{L}, \sigma \in \mathcal{K}$ and $q>0$. Due to compactness of the sets $\tilde{\mathcal{W}}$ and $\mathcal{W}_{y}$, additional bounds can be given to relate the set point distance from both:

$$
\begin{gathered}
|y|_{\tilde{\mathcal{W}}} \leq \rho_{3}\left(|y|_{\mathcal{W}_{y}}\right)+b_{3} \\
|y|_{\mathcal{W}_{y}} \leq \rho_{4}\left(|y|_{\tilde{\mathcal{W}}}\right)+b_{4},
\end{gathered}
$$

for some $\rho_{3}, \rho_{4} \in \mathcal{K}_{\infty}$ and $b_{3}, b_{4} \geq 0$. By embedding bounds (A.12) and (A.13) in estimate (A.11), for all $t \geq 0$ it holds:

$$
|Y(t, y ; d)|_{\tilde{\mathcal{W}}} \leq \kappa^{\prime}\left(|y|_{\tilde{\mathcal{W}}}, t\right)+\sigma^{\prime}(\|d\|)+q^{\prime}
$$

with $\kappa^{\prime}:=\rho_{3} \circ \kappa \circ \rho_{4}, \sigma^{\prime}=\rho_{3} \circ \sigma$, and $q^{\prime}:=\rho_{3}(q)+$ $\rho_{3}\left(\kappa\left(b_{4}\right)\right)+b_{3}$. By taking the limit for $t \rightarrow+\infty$, inequality (A.5) immediately follows.

Lemma Appendix A.2. Assume that system (6a) admits an ISS-Lyapunov function, namely conditions (A.1) and (A.2) hold. Suppose that $\tilde{\alpha}_{x}$ is a $\mathcal{K}_{\infty}$ function so that $\tilde{\alpha}_{x}(r)=\mathcal{O}\left(\alpha_{x}(r)\right)$ as $r \rightarrow 0^{+}$. Then there exists a positive-definite and proper function $\tilde{V}_{x}(x)$, a $\mathcal{K}_{\infty}$ function $\tilde{\gamma}_{x}$, and a positive constant $\tilde{q}_{x}$ such that $\dot{\tilde{V}}_{x}(x) \leq$ $-\tilde{\alpha}_{x}\left(|x|_{\mathcal{W}_{x}}\right)+\tilde{\gamma}_{x}(|d|)+\tilde{q}_{x}$

Proof. We introduce a new candidate ISS-Lyapunov function $\tilde{V}_{x}:=\rho \circ V_{x}$ where $\rho$ is a $\mathcal{K}_{\infty}$ function defined in turn by an integral of the form

$$
\rho(s):=\int_{0}^{s} q(t) d t
$$

and where $q$ is a positive, non-decreasing function which will be defined in the following. Due to $\rho$ being a $\mathcal{K}_{\infty}$ function, inequality (A.1) translates to:

$$
\tilde{\alpha}_{x, 1}\left(|x|_{\mathcal{W}_{x}}\right) \leq \tilde{V}_{x}(x) \leq \tilde{\alpha}_{x, 2}\left(|x|_{\mathcal{W}_{x}}\right)+\tilde{c}_{x},
$$

with $\tilde{\alpha}_{x, 1}:=\rho \circ \alpha_{x, 1}, \tilde{\alpha}_{x, 2}:=\rho \circ 2 \alpha_{x, 2}$, and $\tilde{c}_{x}:=\rho\left(2 c_{x}\right)$. From condition (A.2), we have that:

$$
\dot{\tilde{V}}_{x}(x) \leq q\left(V_{x}(x)\right)\left[-\alpha_{x}\left(|x|_{\mathcal{W}_{x}}\right)+\gamma_{x}(|d|)+q_{x}\right] .
$$

The following two cases arises: 
- if $\gamma_{x}(|d|)+q_{x} \leq \frac{1}{2} \alpha_{x}\left(|x|_{\mathcal{W}_{x}}\right)$ then

$$
\begin{aligned}
& q\left(V_{x}(x)\right)\left[\gamma_{x}(|d|)-\alpha_{x}\left(|x|_{\mathcal{W}_{x}}\right)+q_{x}\right] \\
& \quad \leq-\frac{1}{2} q\left(V_{x}(x)\right) \alpha_{x}\left(|x|_{\mathcal{W}_{x}}\right) \\
& \quad \leq-\frac{1}{2} q\left(\alpha_{x, 1}\left(|x|_{\mathcal{W}_{x}}\right)\right) \alpha_{x}\left(|x|_{\mathcal{W}_{x}}\right)
\end{aligned}
$$

- if $\gamma_{x}(|d|)+q_{x} \geq \frac{1}{2} \alpha_{x}\left(|x|_{\mathcal{W}_{x}}\right)$ then

$$
\begin{aligned}
& q\left(V_{x}(x)\right)\left[\gamma_{x}(|d|)+q_{x}\right] \\
& \quad \leq q\left(\alpha_{x, 2}\left(|x|_{\mathcal{W}_{x}}+c_{x}\right)\right)\left[\gamma_{x}(|d|)+q_{x}\right] \\
& \leq q\left(\alpha_{x, 2}\left(2|x|_{\mathcal{W}_{x}}\right)+\alpha_{x, 2}\left(2 c_{x}\right)\right)\left[\gamma_{x}(|d|)+q_{x}\right] \\
& \leq q\left[\alpha_{x, 2}\left(2 \alpha_{x}^{-1}\left(2 \gamma_{x}(|d|)+2 q_{x}\right)\right)+\alpha_{x, 2}\left(2 c_{x}\right)\right] \\
& \quad\left[\gamma_{x}(|d|)+q_{x}\right] \\
& \leq q\left[\alpha_{x, 2}\left(2 \alpha_{x}^{-1}\left(4 \gamma_{x}(|d|)\right)+2 \alpha_{x}^{-1}\left(4 q_{x}\right)\right)+\right. \\
&\left.\quad \alpha_{x, 2}\left(2 c_{x}\right)\right]\left[\gamma_{x}(|d|)+q_{x}\right] \\
& \leq q\left[\alpha_{x, 2}\left(4 \alpha_{x}^{-1}\left(4 \gamma_{x}(|d|)\right)\right)+\alpha_{x, 2}\left(4 \alpha_{x}^{-1}\left(4 q_{x}\right)\right)+\right. \\
&\left.\quad \alpha_{x, 2}\left(2 c_{x}\right)\right]\left[\gamma_{x}(|d|)+q_{x}\right] \\
& \leq q\left(\theta(|d|)+c_{\theta}\right)\left[\gamma_{x}(|d|)+q_{x}\right] \\
& \leq q\left(\theta(|d|)+c_{\theta}\right) \gamma_{x}(|d|)+q_{x} q(2 \theta(|d|))+q_{x} q\left(2 c_{\theta}\right) \\
& \leq \tilde{\gamma}_{x}(|d|)+\tilde{q}_{x}
\end{aligned}
$$

where we have made use of the general formula:

$\alpha(a+b) \leq \alpha(2 a)+\alpha(2 b)$ for all $\alpha \in \mathcal{K}_{\infty}$ and $a, b>0$,

and we have defined:

$$
\begin{aligned}
\theta & :=\alpha_{x, 2} \circ 4 \alpha_{x}^{-1} \circ 4 \gamma_{x} \\
c_{\theta} & :=\alpha_{x, 2}\left(4 \alpha_{x}^{-1}\left(4 q_{x}\right)\right)+\alpha_{x, 2}\left(2 c_{x}\right) \\
\tilde{\gamma}_{x}(r) & :=q\left(\theta(r)+c_{\theta}\right) \gamma_{x}(r)+q_{x} q(2 \theta(r)) \text { for all } r>0 \\
\tilde{q}_{x} & :=q_{x} q\left(2 c_{\theta}\right) .
\end{aligned}
$$

Therefore, inequality (A.17) can be rewritten as:

$$
\dot{\tilde{V}}_{x}(x) \leq-\frac{1}{2} q\left(\alpha_{x, 1}\left(|x|_{\mathcal{W}_{x}}\right)\right) \alpha_{x}\left(|x|_{\mathcal{W}_{x}}\right)+\tilde{\gamma}_{x}(|d|)+\tilde{q}_{x}
$$

By setting $\beta:=\frac{1}{2} \alpha_{x} \circ \alpha_{x, 1}^{-1}$ and $\tilde{\beta}:=\frac{1}{2} \tilde{\alpha}_{x} \circ \alpha_{x, 1}^{-1}$, Lemma 2 in [13] gives us the positive definite and non-decreasing function $q$ such that:

$$
\left(\frac{1}{2} \tilde{\alpha}_{x} \circ \alpha_{x, 1}^{-1}\right)(s) \leq q(s)\left(\frac{1}{2} \alpha_{x} \circ \alpha_{x, 1}^{-1}\right)(s) \text { for all } s>0 .
$$

By replacing $s$ with $\alpha_{x, 1}\left(|x|_{\mathcal{W}_{x}}\right)$ in (A.20), it then follows that:

$$
\dot{\tilde{V}}_{x}(x) \leq-\tilde{\alpha}_{x}\left(|x|_{\mathcal{W}}\right)+\tilde{\gamma}_{x}(|d|)+\tilde{q_{x}} .
$$

Lemma Appendix A.3. Assume that system (6b) admits an ISS-Lyapunov function, namely conditions (A.3) and (A.4) hold. Suppose that $\tilde{\gamma}_{z}$ is a $\mathcal{K}_{\infty}$ function so that $\gamma_{z}(r)=\mathcal{O}\left(\tilde{\gamma}_{z}(r)\right)$ as $r \rightarrow+\infty$. Then there exists a positive-definite and proper function $\tilde{V}_{z}(z), a \mathcal{K}_{\infty}$ function $\tilde{\alpha}_{z}$, and a positive constant $\tilde{q}_{z}$ such that $\dot{\tilde{V}}_{z}(z) \leq$ $-\tilde{\alpha}_{z}\left(|z|_{\mathcal{W}}\right)+\tilde{\gamma}_{z}(|x|)+\tilde{q}_{z}$.
Proof. We introduce a new candidate ISS-Lyapunov function $\tilde{V}_{z}:=\rho \circ V_{x}$ with $\rho$ being a $\mathcal{K}_{\infty}$ function as defined in (A.15), where $q$ is a positive, non-decreasing function which will be defined in the following. In a similar way as in the proof of Lemma Appendix A.2, inequality (A.4) is bounded from above by:

$$
\begin{aligned}
\dot{\tilde{V}}_{z}(z) \leq- & \frac{1}{2} q\left(\alpha_{z, 1}\left(|z| \mathcal{W}_{z}\right)\right) \alpha_{z}\left(|z|_{\mathcal{W}_{z}}\right) \\
& +q\left(\theta(|x|)+c_{\theta}\right)\left[\gamma_{z}(|x|)+q_{z}\right]
\end{aligned}
$$

where we have defined:

$$
\begin{aligned}
\theta & :=\alpha_{z, 2} \circ 4 \alpha_{z}^{-1} \circ 4 \gamma_{z} \\
c_{\theta} & :=\alpha_{z, 2}\left(4 \alpha_{z}^{-1}\left(4 q_{z}\right)\right)+\alpha_{z, 2}\left(2 c_{z}\right) .
\end{aligned}
$$

By setting $\beta:=\gamma_{z} \circ \theta^{-1}$ and $\tilde{\beta}:=\tilde{\gamma}_{z} \circ \theta^{-1}$, Lemma Appendix A.4 gives us the positive definite and non-decreasing function $q$ such that:

$q\left(s+c_{\theta}\right)\left(\gamma_{z}\left(\theta^{-1}(s)\right)+q_{z}\right) \leq \tilde{\gamma}_{z}\left(\theta^{-1}(s)\right)+\tilde{q}_{z}$ for all $s>0$.

By replacing $s$ with $\theta(|x|)$, we obtain:

$$
q\left(\theta(|x|)+c_{\theta}\right)\left(\gamma_{z}(|x|)+q_{z}\right) \leq \tilde{\gamma}_{z}(|x|)+\tilde{q}_{z} \text { for all } s>0 .
$$

Therefore, inequality (A.21) reads as:

$$
\dot{\tilde{V}}_{z}(z) \leq-\tilde{\alpha}_{z}\left(|z|_{\mathcal{W}_{z}}\right)+\tilde{\gamma}_{z}(|x|)+\tilde{q}_{z},
$$

with $\tilde{\alpha}_{z}:=\frac{1}{2} q\left(\alpha_{z, 1}\left(|z|_{\mathcal{W}_{z}}\right) \alpha_{z}\left(|z|_{\mathcal{W}_{z}}\right)\right)$.

Lemma Appendix A.4. Assume that the functions $\beta, \tilde{\beta} \in$ $\mathcal{K}_{\infty}$ are such that $\beta(r)=\mathcal{O}(\tilde{\beta}(r))$ as $r \rightarrow+\infty$. Then, for all $q_{x}, \tilde{q}_{x}, c_{\theta}>0$, there exists a positive non-decreasing function $q: \mathbb{R}_{>0} \rightarrow \mathbb{R}_{>0}$ so that

$$
q\left(r+c_{\theta}\right)\left[\beta(r)+q_{z}\right] \leq \tilde{\beta}(r)+\tilde{q}_{z} .
$$

Proof. Due to $\beta(r)=\mathcal{O}(\tilde{\beta}(r))$, the function

$$
\frac{\tilde{\beta}(r)+\tilde{q}_{z}}{\beta(r)+q_{z}}
$$

is well-defined and continuous for $r>0$ and it is bounded below by a strictly positive number on any interval of the form $\left[r_{0},+\infty\right)$ with $r_{0} \geq 0$. Then, the function

$$
\tilde{q}(s):=\inf _{r \geq s} \frac{\tilde{\beta}(r)+\tilde{q}_{z}}{\beta(r)+q_{z}}
$$

satisfies the property $\tilde{q}(0)>0$. We define the following function on the interval $\left[c_{\theta},+\infty\right)$ as follows:

$$
q(r):=\tilde{q}\left(r-c_{\theta}\right) \text { if } r \geq c_{\theta} .
$$

It then follows that $q\left(r+c_{\theta}\right)=\tilde{q}(r) \forall r \geq 0$ and, moreover, property (A.24) holds for all $r \geq 0$. 


\section{References}

[1] Angeli, D., Mar 2002. A Lyapunov approach to incremental stability properties. Automatic Control, IEEE Transactions on 47 (3), 410-421.

[2] Angeli, D., Efimov, D., 2015. Characterizations of input-to-state stability for systems with multiple invariant sets. Automatic Control, IEEE Transactions on PP (99), 1-1.

[3] Bathia, N. P., Szegö, G. P., 1970. Stability theory of dynamical systems. Springer-Verlag, Berlin.

[4] Besicovich, A. S., 1954. Almost periodic functions. Dover Publications, Inc., New York.

[5] Forni, P., Angeli, D., 2015. Input-to-state stability for cascade systems with decomposable invariant sets. In: Decision and Control (CDC), 2015 IEEE 54nd Annual Conference on. Submitted.

[6] Ijspeert, A. J., 2008. Central pattern generators for locomotion control in animals and robots: A review. Neural Networks 21 (4), $642-653$, robotics and Neuroscience.

[7] Kim, S.-J., 2005. A note on the connection between incremental input-to-state stability and fading memory in nonlinear systems.

[8] Mischaikow, K., Smith, H., Thieme, H. R., 1995. Asymptotically autonomous semiflows: chain recurrence and Lyapunov functions. Transactions of the American Mathematical Society 347 (5), pp. 1669-1685.

[9] Rüffer, B. S., van de Wouw, N., Mueller, M., 2013. Convergent systems vs. incremental stability. Systems \& Control Letters $62(3), 277-285$.

[10] Ryan, E. P., Sontag, E. D., 2006. Well-defined steady-state response does not imply $\{$ CICS $\}$. Systems \& Control Letters $55(9), 707-710$.

[11] Sandberg, I., Jul 1992. Approximately-finite memory and inputoutput maps. Circuits and Systems I: Fundamental Theory and Applications, IEEE Transactions on 39 (7), 549-556.

[12] Sepulchre, R., Jankovic, M., Kokotovic, P., 1997. Constructive nonlinear control. Springer-Verlag.

[13] Sontag, E., Teel, A., Aug 1995. Changing supply functions in input/state stable systems. Automatic Control, IEEE Transactions on 40 (8), 1476-1478.

[14] Sontag, E. D., Feb 2003. A remark on the converging-input converging-state property. IEEE Transactions on Automatic Control 48 (2), 313-314.

[15] Sontag, E. D., 2006. Input to state stability: Basic concepts and results. In: Nonlinear and Optimal Control Theory. Springer, pp. $163-220$.

[16] Thieme, H., 1992. Convergence results and a PoincaréBendixson trichotomy for asymptotically autonomous differential equations. Journal of Mathematical Biology 30 (7), 755-763. 